\title{
Ground state solutions for fractional Schrödinger equation with variable potential and Berestycki-Lions type nonlinearity
}

Jing Chen ${ }^{1 *}$ (D) and Zu Gao ${ }^{2}$

"Correspondence:

cjhnust@aliyun.com

'School of Mathematics and

Computing Sciences, Hunan

University of Science and

Technology, Xiangtan, P.R. China

Full list of author information is

available at the end of the article

\begin{abstract}
We consider the following nonlinear fractional Schrödinger equation:

$$
(-\triangle)^{s} u+V(x) u=g(u) \text { in } \mathbb{R}^{N},
$$

where $s \in(0,1), N>2 s, V(x)$ is differentiable, and $g \in C^{\prime}(\mathbb{R}, \mathbb{R})$. By exploiting the minimization method with a constraint over Pohožaev manifold, we obtain the existence of ground state solutions. With the help of Pohožaev identity we also process the existence of the least energy solutions for the above equation. Our results improve the existing study on this nonlocal problem with Berestycki-Lions type nonlinearity to the one that does not need the oddness assumption
\end{abstract}

MSC: $35 \mathrm{~A} 15 ; 35 J 60$

Keywords: Fractional Schrödinger equation; Pohožaev manifold; Ground state solutions; Least energy solution

\section{Introduction}

In the present paper, we investigate the following fractional Schrödinger equation:

$$
(-\triangle)^{s} u+V(x) u=g(u) \quad \text { in } \mathbb{R}^{N},
$$

where $s \in(0,1), N>2 s,(-\triangle)^{s}$ is fractional Laplacian that is the pseudodifferential operator with symbol $|\xi|^{s}$, i.e.,

$$
(-\triangle)^{s} u=\mathcal{F}^{-1}\left(|\xi|^{2 s} \mathcal{F} u\right),
$$

where $\mathcal{F}$ is the Fourier transform in $\mathbb{R}^{N}$. This type of fractional scalar field equation with an external potential appears in many models in mathematical physics, especially in the study of particles on stochastic fields modeled by Lévy processes, see [24]. Problems with fractional Laplacian have attracted much attention in recent years; they model a wide class of problems arising in applications, such as turbulence and water wave, anomalous dynamics, flames propagation and chemical reactions in liquids, population dynamics, geophysical fluid dynamics and finance, we refer the reader, for instance, to $[5,13,15$,

(c) The Author(s) 2019. This article is distributed under the terms of the Creative Commons Attribution 4.0 International License (http://creativecommons.org/licenses/by/4.0/), which permits unrestricted use, distribution, and reproduction in any medium, provided you give appropriate credit to the original author(s) and the source, provide a link to the Creative Commons license, and indicate if changes were made. 
$16,19,23,30,31$ ] and the related recent references [37-40]. We recall that $u(x)$ is said to be a least energy solution (or ground state) of (1.1) if and only if $I(u)=m_{0}$, where $m_{0}:=\inf \left\{I(w): w \in H^{s}\left(\mathbb{R}^{N}\right) \backslash\{0\}\right.$ is a solution of (1.1) $\}$. Here, $I$ is the energy functional corresponding to (1.1) defined on fractional Sobolev space $H^{s}\left(\mathbb{R}^{N}\right)$ :

$$
I(u)=\frac{1}{2} \int_{\mathbb{R}^{N}}|\xi|^{2 s}|\hat{u}|^{2} \mathrm{~d} \xi+\frac{1}{2} \int_{\mathbb{R}^{N}} V(x) u^{2} \mathrm{~d} x-\int_{\mathbb{R}^{N}} G(u) \mathrm{d} x,
$$

where $\hat{u}=\mathcal{F} u$ and $G(u)=\int_{0}^{u} g(\zeta) \mathrm{d} \zeta$.

If the potential is a positive constant, for convenience, let $V(x) \equiv V_{\infty}$, then (1.1) reduces to the following autonomous form:

$$
(-\triangle)^{s} u+V_{\infty} u=g(u) \quad \text { in } \mathbb{R}^{N}
$$

its energy functional is as follows:

$$
I^{\infty}(u)=\frac{1}{2} \int_{\mathbb{R}^{N}}|\xi|^{2 s}|\hat{u}|^{2} \mathrm{~d} \xi+\frac{V_{\infty}}{2} \int_{\mathbb{R}^{N}} u^{2} \mathrm{~d} x-\int_{\mathbb{R}^{N}} G(u) \mathrm{d} x .
$$

During the past years there has been an increasing interest in the existence and properties of ground state for Problem (1.3). In the very particular case of $V_{\infty}=1$, when $g(u)=$ $u^{\alpha+1}$, Frank and Lenzmann [16] established the uniqueness and nondegeneracy of ground state in $\mathbb{R}$ for (1.3), that is, $N=1$, when $\alpha \in\left(0, \frac{4 s}{1-2 s}\right)$ for $s \in(0,1 / 2)$ and $\alpha \in(0,+\infty)$ for $s \in[1 / 2,1)$ by the separation of eigenvalues of the linearized operator. Fall and Valdinoci [14] later on extended the result of [16] in any dimension when $s$ is sufficiently close to 1 . Furthermore, Frank, Lenzmann, and Silvestre [17] extended the results of [16] to general $s \in(0,1)$. In the same spirit of [29] devised by Rabinowitz for $s=1$, Felmer, Quaas, and Tan [15] obtained the existence of classical positive solution for (1.3) and proved that $I^{\infty}$ has a critical point of mountain-pass type. It is worth noticing that they needed the classic Nehari monotonic condition and the Ambrosetti-Rabinowitz condition to obtain the compactness. When $g(u)=|u|^{p-1} u, p \in\left(1, \frac{N+2 s}{N-2 s}\right)$, Dipierro, Palatucci, and Valdinoci [13] exploited the constrained minimization method developed by Berestycki and Lions in [3] to prove the existence of nontrivial radial symmetric solutions for (1.3). They considered the following variational problem:

$$
\min \left\{[u]_{H^{s}\left(\mathbb{R}^{N}\right)}^{2}: u \in H^{s}\left(\mathbb{R}^{N}\right), \int_{\mathbb{R}^{N}} G(u) \mathrm{d} x=1\right\}
$$

where $[u]_{H^{s}\left(\mathbb{R}^{N}\right)}$ is defined in Sect. 2. By using the fractional Polya-Szegö inequality (see [28], Theorem 1.1), they obtained the minimizing sequence, which can be selected as a sequence of nonnegative, spherically symmetric, and decreasing functions in $L^{2}\left(\mathbb{R}^{N}\right)$. Then, by using a radial lemma combined with a compactness lemma due to Strauss (see [3], Theorem A.I), they concluded that the limit $\bar{u}$ solves the minimization problem (1.5). When $V_{\infty}=0$, Chang and Wang [7] considered an extension of the equation in (1.3) by replacing the nonlinearity $|u|^{p-1} u$ with a wide class of odd differentiable functions $g(u)$ satisfying the following conditions:

(G0) $g(0)=0$; 
(G1) $g \in C^{1}(\mathbb{R}, \mathbb{R})$ and there exists a constant $C_{0}>0$ such that

$$
|g(u)| \leq C_{0}\left(1+|u|^{2_{s}^{*}-1}\right), \quad \forall u \in \mathbb{R},
$$

where $2_{s}^{*}:=\frac{2 N}{N-2 s} ;$

(G2) $\lim _{u \rightarrow 0} \frac{g(u)}{u}=0$;

(G3) $\lim _{|u| \rightarrow+\infty} \frac{|g(u)|}{|u|^{2 *-1}}=0$;

(G4) there exists $u_{0}>0$ such that $G\left(u_{0}\right)>\frac{1}{2} V_{\infty} u_{0}^{2}$.

With the help of the following Pohožaev identity for (1.3):

$$
\mathcal{P}^{\infty}(u):=\frac{N-2 s}{2} \int_{\mathbb{R}^{N}}|\xi|^{2 s}|\hat{u}|^{2} \mathrm{~d} \xi+\frac{N}{2} V_{\infty} \int_{\mathbb{R}^{N}} u^{2} \mathrm{~d} x-N \int_{\mathbb{R}^{N}} G(u) \mathrm{d} x=0,
$$

under conditions $(\mathrm{G} 0)-(\mathrm{G} 4)\left(\right.$ when $2_{s}^{*}$ is replaced by $2^{*}=2 N /(N-2),(\mathrm{G} 0)-(\mathrm{G} 4)$ are classical Berestycki-Lions conditions), they obtained a positive ground state solution of (1.3). Different from the classical local counterpart, it requires that $g \in C^{1}(\mathbb{R}, \mathbb{R})$ in [7] to guarantee that the $s$-harmonic extension $E_{s}(u)$ is smooth enough for general $s \in(0,1)$. The $s$-harmonic extension technique is so important in the proof of Pohožaev identity for (1.3) which was introduced by Caffarelli and Silvestre in [5] that it can transform the nonlocal problem to a local one via the Dirichlet-Neumann map. In fact, the smoothness of $g$ only is needed to prove such an identity for (1.3). Without the symmetric radial decreasing rearrangement, Chang and Wang applied cut-offs and a helpful analog result of Strauss's compactness lemma to recover the compactness, which is still applicable when the property of uniform decay at infinity of bounded (PS) sequences is not available.

When $V$ is non-constant and $s=1,(1.1)$ reduces to the following classic nonlinear Schrödinger equation:

$$
-\triangle u+V(x) u=g(u) \quad \text { in } \mathbb{R}^{N} .
$$

There are many studies on Schrödinger equations and related problems under the Berestycki-Lions assumptions, see for example [1, 8-11, 21, 29, 34-36]. Following the ideas of [1], Secchi [30] investigated (1.1), where $g$ satisfies (G0)-(G4) and $V$ does the following conditions:

( $\left.\mathrm{VO}^{\prime}\right) \lim _{|x| \rightarrow \infty} V(x)=0$ and $V(x)=V(|x|)$;

(V1) $V \in C^{1}\left(\mathbb{R}^{N},[0, \infty)\right)$;

(V2) $\|\max \{\nabla V(x) \cdot x, 0\}\|_{L^{\frac{N}{2 s}\left(\mathbb{R}^{N}\right)}}<2 s S_{s}$, where $\cdot$ denotes the inner product in $\mathbb{R}^{N}$ and $S_{s}$ will be defined in Sect. 2.

Indeed, (V2) is helpful for getting the boundedness of the (PS) sequence by using monotonicity trick. Applying the compactness lemma in fractional Sobolev radial space $H_{\text {rad }}^{s}\left(\mathbb{R}^{N}\right)$ together with Jeanjean's monotonicity trick, Secchi obtained the existence of radially symmetric solutions for (1.1). Moreover, making use of the idea from [16], Secchi proved that each solution of (1.1) satisfies the following Pohožaev type identity:

$$
\begin{aligned}
\mathcal{P}(u):= & \frac{N-2 s}{2} \int_{\mathbb{R}^{N}}|\xi|^{2 s}|\hat{u}|^{2} \mathrm{~d} \xi+\frac{1}{2} \int_{\mathbb{R}^{N}}[N V(x)+\nabla V(x) \cdot x] u^{2} \mathrm{~d} x \\
& -N \int_{\mathbb{R}^{N}} G(u) \mathrm{d} x=0 .
\end{aligned}
$$


For the nonradial cases, by using Pohožaev identity and Jeanjean's monotonicity trick, Chang investigated (1.1) in [19] and obtained the existence of ground state solutions when $V$ satisfied (V1), (V2), and the following unbounded condition:

$\left(\mathrm{VO}^{\prime \prime}\right)$ There exists $r>0$ such that, for any $M>0$,

$$
\operatorname{mes}\left(\left\{x \in B_{r}(y): V(x) \leq M\right\}\right) \rightarrow 0 \quad \text { as }|y| \rightarrow \infty
$$

At the same time, $g$ is assumed as a $C^{1}$ function satisfying (G0), (G2), (G3), and the following geometry conditions:

(G5) There exists $\beta_{0} \in(0,+\infty)$ such that

$$
-\beta_{0} \leq \liminf _{t \rightarrow 0^{+}} \frac{2 G(t)}{t^{2}} \leq \limsup _{t \rightarrow 0^{+}} \frac{2 G(t)}{t^{2}}<\inf \alpha_{0}
$$

where $\alpha_{0}$ denotes the spectrum of the operator $(-\triangle)^{s}+V(x): H^{s}\left(\mathbb{R}^{N}\right) \rightarrow H^{-s}\left(\mathbb{R}^{N}\right)$ and

$$
\inf \alpha_{0}=\inf _{\substack{u \in H^{s}\left(\mathbb{R}^{N}\right) \\ u \neq 0}} \frac{\int_{\mathbb{R}^{N}}|\xi|^{2 s}|\hat{u}|^{2} \mathrm{~d} \xi+\int_{\mathbb{R}^{N}} V(x) u^{2} \mathrm{~d} x}{\int_{\mathbb{R}^{N}} u^{2} \mathrm{~d} x} ;
$$

(G6) $\liminf _{t \rightarrow+\infty} \frac{2 G(t)}{t^{2}}>\inf \alpha_{0}$.

Clearly, in Chang's result neither the superlinear growth nor the asymptotically linear growth is required. For the case of the asymptotically linear growth on $g$, we refer to [6].

In their recent paper [25], Liu and Ouyang considered (1.1) and obtained the existence of positive ground states for (1.1) with a general potential and a nonnegative nonlinearity. Specifically, their results need conditions (G0)-(G4), (V1), (V2), and the following condition on $V$ :

(V3) $V(x) \leq V_{\infty}:=\lim _{|y| \rightarrow \infty} V(y)$ for all $x \in \mathbb{R}^{N}$.

Especially, (G0) is necessary to their results for Schwarz spherical rearrangement technique used in [25] to recover compactness.

Thanks to the above work, the aim of this paper is to study Problem (1.1) with a general potential by preserving the Berestycki-Lions conditions on $g$; however, the positive definiteness condition (G0) is not necessary. Motivated by [18, 32, 33, 35], we shall exploit a nontrivial approach (called the least energy squeeze approach in [35]) to show (1.1) has a solution $\bar{u} \in \mathcal{M}$ such that

$$
I(\bar{u})=\min _{u \in \mathcal{M}} I(u), \quad \mathcal{M}:=\left\{u \in H^{s}\left(\mathbb{R}^{N}\right) \backslash\{0\}: \mathcal{P}(u)=0\right\}
$$

under (G1)-(G4), (V1), (V3), and an additional decay condition on $V$ in place of (V2):

(V4) There exists $\theta \in[0,1)$ such that

$$
\begin{aligned}
& N[V(x)-V(t x)]+[\nabla V(x) \cdot x-\nabla V(t x) \cdot t x]+\frac{K(s)(N-2 s) \theta}{t^{2 s}|x|^{2 s}}\left(t^{2 s}-1\right) \\
& \begin{cases}\geq 0, & t \in[1,+\infty), \\
\leq 0, & t \in(0,1),\end{cases}
\end{aligned}
$$

where $K(s):=\frac{2^{2 s} \Gamma^{2}\left(\frac{N+2 s}{4}\right)}{\Gamma^{2}\left(\frac{N-2 s}{4}\right)}$ and $\Gamma$ is the gamma function. 
Our main result about the existence of ground state solution of Pohožaev type is illustrated as follows.

Theorem 1.1 Suppose that $V$ and $g$ satisfy (V1), (V3), (V4), and (G1)-(G4). Then Problem (1.1) has a solution $\bar{u} \in H^{s}\left(\mathbb{R}^{N}\right)$ such that $I(\bar{u})=\inf _{\mathcal{M}} I>0$.

Set

$$
\mathcal{M}^{\infty}:=\left\{u \in H^{s}\left(\mathbb{R}^{N}\right) \backslash\{0\}: \mathcal{P}^{\infty}(u)=0\right\},
$$

we are now in a position to state the result in the case of constant potential.

Theorem 1.2 Suppose that $g$ satisfies (G1)-(G4). Then Problem (1.3) has a solution $\bar{u} \in$ $H^{s}\left(\mathbb{R}^{N}\right)$ such that $I^{\infty}(\bar{u})=\inf _{\mathcal{M}} I^{\infty}>0$.

The proof of Theorem 1.1 comes from the idea in $[25,35]$. To be specific, we employ the minimization method with a natural constraint for $I$ over Pohožaev manifold $\mathcal{M}$ to find a ground state solution for (1.1) without the requirement for radial compactness or other symmetry property on $V$. By the standard steps, firstly, we choose a minimizing sequence $\left\{u_{n}\right\} \in \mathcal{M}$ and show that $\left\{u_{n}\right\}$ is bounded in $H^{s}\left(\mathbb{R}^{N}\right)$. Subsequently, by the concentration compactness principle, we prove $\left\{u_{n}\right\}$ converge to $\bar{u} \in H^{s}\left(\mathbb{R}^{N}\right) \backslash\{0\}$. For the purpose of proving the minimizing problem (1.9) is solvable, we make some translation and scale changes to the minimizing subsequence before a crucial inequality related to $I(u), I\left(u_{t}\right)$, and $\mathcal{P}(u)$ (see Lemma 3.2) is used to recover the compactness, where $u_{t}(x)=u(x / t)$. Note that the inequality takes place in this paper more than once, especially the significance of the inequality in this step lies in the fact that it enables us to keep away from the Schwarz spherical rearrangement, which plays an important role in [25], and handle the case when the information of $I^{\prime}\left(u_{n}\right)$ is not clear. In the end, we show that the minimizer $\bar{u}$ is a critical point of $I$ by a deformation lemma.

In the rest of this paper, based on Theorem 1.2, we investigate the existence of the least energy solutions for (1.1) under (G1)-(G4). In this situation, we use the following weak decay assumption on $\nabla V$ instead of (V4):

(V5) There exists $\hat{\theta} \in[0, s)$ such that

$$
\nabla V(x) \cdot x \leq \frac{2 K(s) \hat{\theta}}{|x|^{2 s}}, \quad x \in \mathbb{R}^{N} \backslash\{0\}
$$

More precisely, we have the following theorem.

Theorem 1.3 Suppose that $V$ and $g$ satisfy (V1), (V3), (V5), and (G1)-(G4). Then Problem (1.1) has a least energy solution.

To prove Theorem 1.3, we consider a family of perturbed functionals $I_{\lambda}: H^{s}\left(\mathbb{R}^{N}\right) \rightarrow \mathbb{R}$ defined by

$$
I_{\lambda}(u)=\frac{1}{2} \int_{\mathbb{R}^{N}}|\xi|^{2 s}|\hat{u}|^{2} \mathrm{~d} \xi+\frac{1}{2} \int_{\mathbb{R}^{N}} V(x) u^{2} \mathrm{~d} x-\lambda \int_{\mathbb{R}^{N}} G(u) \mathrm{d} x
$$


for $\lambda$ near 1 . These functionals have the mountain pass geometry, and the corresponding mountain pass levels are denoted by $c_{\lambda}$. Corresponding to (1.10), we let

$$
I_{\lambda}^{\infty}(u)=\frac{1}{2} \int_{\mathbb{R}^{N}}|\xi|^{2 s}|\hat{u}|^{2} \mathrm{~d} \xi+\frac{1}{2} \int_{\mathbb{R}^{N}} V_{\infty} u^{2} \mathrm{~d} x-\lambda \int_{\mathbb{R}^{N}} G(u) \mathrm{d} x .
$$

As a consequence of Theorem 1.2, for each $\lambda \in[0.5,1]$, there exists a minimizer $u_{\lambda}^{\infty}$ of $I_{\lambda}^{\infty}$ on $\mathcal{M}_{\lambda}^{\infty}$, where

$$
\mathcal{M}_{\lambda}^{\infty}:=\left\{u \in H^{s}\left(\mathbb{R}^{N}\right) \backslash\{0\}: \mathcal{P}_{\lambda}^{\infty}(u)=0\right\}
$$

and

$$
\mathcal{P}_{\lambda}^{\infty}(u):=\frac{N-2 s}{2} \int_{\mathbb{R}^{N}}|\xi|^{2 s}|\hat{u}|^{2} \mathrm{~d} \xi+\frac{N}{2} \int_{\mathbb{R}^{N}} V_{\infty} u^{2} \mathrm{~d} x-N \lambda \int_{\mathbb{R}^{N}} G(u) \mathrm{d} x .
$$

In this part, we use the same trick as that in $[32,35]$ to recover the global compactness after a bounded (PS) sequence $\left\{u_{n}(\lambda)\right\} \subset H^{s}\left(\mathbb{R}^{N}\right)$ at level $c_{\lambda}$ is found. We deduce the following relationship between $c_{\lambda}$ and $m_{\lambda}^{\infty}:=\inf _{\mathcal{M}_{\lambda}^{\infty}} I_{\lambda}^{\infty}$, that is, there exists $\bar{\lambda} \in[0.5,1)$ such that

$$
c_{\lambda}<m_{\lambda}^{\infty}, \quad \lambda \in(\bar{\lambda}, 1]
$$

Next, making use of (1.13) combined with a decomposition of bounded (PS)-sequence in $[25,26]$, we can get a nontrivial critical point $u_{\lambda}$ of $I_{\lambda}$ which possesses energy $c_{\lambda}$ for almost every $\lambda \in(\bar{\lambda}, 1]$. Finally, we prove that (1.1) processes a least energy solution with the help of Pohožaev identity.

To illustrate conveniently, we introduce some useful notation here. In the sequel, $\|u\|_{p}=$ $\left(\int_{\mathbb{R}^{N}}|u|^{p} \mathrm{~d} x\right)^{1 / p}$ denotes the norm of the Lebesgue space $L^{p}\left(\mathbb{R}^{N}\right)(1 \leq p \leq \infty)$. For $x \in \mathbb{R}^{N}$ and $r>0, B_{r}(x):=\left\{y \in \mathbb{R}^{N}:|y-x|<r\right\}$. Throughout the paper, $C_{1}, C_{2}, \ldots$ denote various positive constants.

The paper is organized as follows. In Sect. 2, we give some preliminaries. In Sect. 3, we give the proof of Theorem 1.1. The limiting equation is studied beforehand and the proof of Theorem 1.2 is also given. Section 4 deals with a least energy solution for (1.1), and the proof of Theorem 1.3 will be given in this section.

\section{Preliminary and variational setting}

The fractional Sobolev space $H^{s}\left(\mathbb{R}^{N}\right)$ and the norm are defined by

$$
H^{s}\left(\mathbb{R}^{N}\right):=\left\{u \in L^{2}\left(\mathbb{R}^{N}\right): \int_{\mathbb{R}^{N}}\left(|\xi|^{2 s}|\hat{u}|^{2}+|\hat{u}|^{2}\right) \mathrm{d} \xi<\infty\right\}
$$

and

$$
\|u\|_{H^{s}\left(\mathbb{R}^{N}\right)}=\left(\int_{\mathbb{R}^{N}}\left(|\xi|^{2 s}|\hat{u}|^{2}+|\hat{u}|^{2}\right) \mathrm{d} \xi\right)^{\frac{1}{2}}
$$

It can be proved (see [12, Proposition 3.4, Proposition 3.6]) that

$$
[u]_{H^{s}\left(\mathbb{R}^{N}\right)}^{2}=2 C(N, s)^{-1} \int_{\mathbb{R}^{N}}|\xi|^{2 s}|\hat{u}|^{2} \mathrm{~d} \xi
$$


and

$$
[u]_{H^{s}\left(\mathbb{R}^{N}\right)}^{2}=2 C(N, s)^{-1}\left\|(-\triangle)^{\frac{s}{2}} u\right\|_{2}^{2},
$$

where $[u]_{H^{s}\left(\mathbb{R}^{N}\right)}$ denotes Gagliarodo (semi)norm of $u$ and $C(N, s)=\left(\int_{\mathbb{R}^{N}} \frac{1-\cos \zeta 1}{|\zeta|^{N+2 s}} \mathrm{~d} \zeta\right)^{-1}$. As a consequence, we have

$$
\int_{\mathbb{R}^{N}}\left|(-\triangle)^{\frac{s}{2}} u\right|^{2} \mathrm{~d} x=\int_{\mathbb{R}^{N}}\left(|\xi|^{s}|\hat{u}|\right)^{2} \mathrm{~d} \xi
$$

and the norm on $H^{s}\left(\mathbb{R}^{N}\right)$ can be written as follows:

$$
\|u\|_{H^{s}\left(\mathbb{R}^{N}\right)}:=\left(\int_{\mathbb{R}^{N}}|u|^{2} \mathrm{~d} x+\int_{\mathbb{R}^{N}}\left|(-\triangle)^{\frac{s}{2}} u\right|^{2} \mathrm{~d} x\right)^{\frac{1}{2}} .
$$

$H^{s}\left(\mathbb{R}^{N}\right)$ is the completion of $C_{0}^{\infty}\left(\mathbb{R}^{N}\right)$ with $\|\cdot\|_{H^{s}\left(\mathbb{R}^{N}\right)}$ and it is continuously embedded into $L^{q}\left(\mathbb{R}^{N}\right)$ for any $q \in\left[2,2_{s}^{*}\right]$, and compactly embedded into $L_{\text {loc }}^{q}\left(\mathbb{R}^{N}\right)$ for any $q \in\left[2,2_{s}^{*}\right)$. Moreover, the best embedding constant is $S_{s}$ defined as

$$
S_{s}:=\inf _{\substack{u \in D^{s, 2}\left(\mathbb{R}^{N}\right) \\ u \neq 0}} \frac{\int_{\mathbb{R}^{N}}\left|(-\Delta)^{\frac{s}{2}} u\right|^{2} \mathrm{~d} x}{\left(\int_{\mathbb{R}^{N}}|u|^{2 *} \mathrm{~d} x\right)^{2 / 2_{s}^{*}}},
$$

where $D^{s, 2}\left(\mathbb{R}^{N}\right)$ denotes homogeneous Sobolev space defined by

$$
D^{s, 2}\left(\mathbb{R}^{N}\right):=\left\{u \in L^{2_{s}^{*}}\left(\mathbb{R}^{N}\right):|\xi|^{s}|\hat{u}| \in L^{2}\left(\mathbb{R}^{N}\right)\right\},
$$

which is also the completion of $C_{0}^{\infty}\left(\mathbb{R}^{N}\right)$ under the norm $\|u\|_{D^{s, 2}\left(\mathbb{R}^{N)}\right)}:=\left(\int_{\mathbb{R}^{N}}\left|(-\triangle)^{\frac{s}{2}} u\right|^{2}\right)^{1 / 2}=$ $\left\|(-\triangle)^{\frac{s}{2}} u\right\|_{2}$. For simplicity, we denote $\|\cdot\|_{H^{s}\left(\mathbb{R}^{N}\right)}$ by $\|\cdot\|$ afterwards.

The following lemma is a fractional version of Lion's vanishing lemma, which is the major tool in variational method in our forthcoming arguments.

Lemma 2.1 ([31]) Assume that $\left\{u_{n}\right\}_{n \in \mathbb{N}}$ is bounded in $H^{s}\left(\mathbb{R}^{N}\right)$ and

$$
\lim _{n \rightarrow \infty} \sup _{y \in \mathbb{R}^{N}} \int_{B_{r}(y)}\left|u_{n}\right|^{2} \mathrm{~d} x=0
$$

for some $r>0$. Then $u_{n} \rightarrow 0$ in $L^{p}\left(\mathbb{R}^{N}\right)$ for all $p \in\left(2,2_{s}^{*}\right)$.

We introduce the following modified version of the monotonicity trick of [20] developed by Jeanjean, which is exploited in constructing bounded (PS) sequences for $I_{\lambda}$ defined in (1.10) for almost every $\lambda$ close to 1 to find the least energy solution.

Proposition 2.2 ([21]) Let $X$ be a Banach space, $\Lambda \subset[0,+\infty)$ be an interval, and

$$
\Phi_{\lambda}(u)=A(u)-\lambda B(u), \quad \forall \lambda \in \Lambda,
$$


(i) either $A(u) \rightarrow+\infty$ or $B(u) \rightarrow+\infty$, as $\|u\| \rightarrow+\infty$;

(ii) $B$ maps every bounded set of $X$ into a set of $\mathbb{R}$ bounded below;

(iii) there are two points $v_{1}, v_{2}$ in $X$ such that

$$
c_{\lambda}:=\inf _{\gamma \in \tilde{\Gamma}} \max _{t \in[0,1]} \Phi_{\lambda}(\gamma(t))>\max \left\{\Phi_{\lambda}\left(v_{1}\right), \Phi_{\lambda}\left(v_{2}\right)\right\}
$$

where

$$
\widetilde{\Gamma}=\left\{\gamma \in C([0,1], X): \gamma(0)=v_{1}, \gamma(1)=v_{2}\right\}
$$

Then, for almost every $\lambda \in \Lambda$, there is a bounded sequence $\left\{u_{n}(\lambda)\right\} \subset X$ such that

(i) $\left\{u_{n}(\lambda)\right\}$ is bounded;

(ii) $\Phi_{\lambda}\left(u_{n}(\lambda)\right) \rightarrow c_{\lambda}$;

(iii) $\Phi_{\lambda}^{\prime}\left(u_{n}(\lambda)\right) \rightarrow 0$ in $X^{*}$, where $X^{*}$ is the dual of $X$.

In our situation, we assume

$$
A(u)=\frac{1}{2} \int_{\mathbb{R}^{N}}\left|(-\triangle)^{\frac{s}{2}} u\right|^{2} \mathrm{~d} x+\frac{1}{2} \int_{\mathbb{R}^{N}} V(x) u^{2} \mathrm{~d} x, \quad B(u)=\frac{1}{2} \int_{\mathbb{R}^{N}} G(u) \mathrm{d} x .
$$

Then $I_{\lambda}(u)=A(u)-\lambda B(u)$. Here, different from [19, 25, 30], $B(u)$ is indefinite sign, so that Jeanjean's monotonicity trick [20] is not applicable. But using an idea from [22], for almost every $\lambda \in[0.5,1]$, we still can obtain that $\left\{u_{n}(\lambda)\right\} \subset H^{s}\left(\mathbb{R}^{N}\right)$ at level $c_{\lambda}$ is a bounded (PS)sequence, because $I_{\lambda}$ has mountain pass geometry almost everywhere.

\section{Existence of ground state solutions of Pohožaev type for (1.1)}

In this section, through discussing the corresponding limit equation (1.3), we will get the proof of Theorem 1.1. Since $V(x) \equiv$ constant which is well covered by (V1), (V3), the following lemmas on $I$ are true for $I^{\infty}$. We begin our arguments with some useful inequalities.

Lemma 3.1 The following inequality holds:

$$
h(t):=2 s-N t^{N-2 s}+(N-2 s) t^{N}>h(1)=0, \quad t \in[0,1) \cup(1,+\infty) .
$$

Moreover, (V4) implies that the following inequality holds:

$$
N t^{N}[V(x)-V(t x)]-\left(1-t^{N}\right) \nabla V(x) \cdot x \geq-\frac{K(s) \theta\left[2 s-N t^{N-2 s}+(N-2 s) t^{N}\right]}{|x|^{2 s}} .
$$

It is easy to verify Lemma 3.1 by a direct calculation.

Lemma 3.2 Suppose that (V1), (V4), (G1)-(G3) hold. Then

$$
\begin{aligned}
I(u) \geq & I\left(u_{t}\right)+\frac{1-t^{N}}{N} \mathcal{P}(u)+\frac{(1-\theta)\left[2 s-N t^{N-2 s}+(N-2 s) t^{N}\right]}{2 N}\left\|(-\triangle)^{\frac{s}{2}} u\right\|_{2}^{2}, \\
& \forall u \in H^{s}\left(\mathbb{R}^{N}\right), t>0 .
\end{aligned}
$$


Proof By the description of fractional Hardy inequality in [4], the authors in [2, 41] obtained the fractional Hardy inequality on the whole $\mathbb{R}^{N}$ named Hardy-Rellich inequality as follows:

$$
\left\|(-\triangle)^{\frac{s}{2}} u\right\|_{2}^{2} \geq 2^{2 s} \frac{\Gamma^{2}\left(\frac{N+2 s}{4}\right)}{\Gamma^{2}\left(\frac{N-2 s}{4}\right)} \int_{\mathbb{R}^{N}} \frac{u^{2}}{|x|^{2 s}} \mathrm{~d} x .
$$

It is obvious that

$$
I\left(u_{t}\right)=\frac{t^{N-2 s}}{2}\left\|(-\triangle)^{\frac{s}{2}} u\right\|_{2}^{2}+\frac{t^{N}}{2} \int_{\mathbb{R}^{N}} V(t x) u^{2} \mathrm{~d} x-t^{N} \int_{\mathbb{R}^{N}} G(u) \mathrm{d} x .
$$

Thus, in view of (V4), (1.2), (3.2), (3.4), and (3.5), we get

$$
\begin{aligned}
I(u)- & I\left(u_{t}\right) \\
= & \frac{1-t^{N-2 s}}{2}\left\|(-\triangle)^{\frac{s}{2}} u\right\|_{2}^{2}+\frac{1}{2} \int_{\mathbb{R}^{N}}\left[V(x)-t^{N} V(t x)\right] u^{2} \mathrm{~d} x-\left(1-t^{N}\right) \int_{\mathbb{R}^{N}} G(u) \mathrm{d} x \\
= & \frac{1-t^{N}}{N}\left\{\frac{N-2 s}{2}\left\|(-\triangle)^{\frac{s}{2}} u\right\|_{2}^{2}+\frac{1}{2} \int_{\mathbb{R}^{N}}[N V(x)+(\nabla V(x), x)] u^{2} \mathrm{~d} x\right. \\
& \left.-N \int_{\mathbb{R}^{N}} G(u) \mathrm{d} x\right\}+\frac{2 s-N t^{N-2 s}+(N-2 s) t^{N}}{2 N}\left\|(-\triangle)^{\frac{s}{2}} u\right\|_{2}^{2} \\
& +\frac{1}{2} \int_{\mathbb{R}^{N}}\left\{t^{N}[V(x)-V(t x)]-\frac{1-t^{N}}{N}(\nabla V(x), x)\right\} u^{2} \mathrm{~d} x \\
\geq & \frac{1-t^{N}}{N} \mathcal{P}(u)+\frac{(1-\theta)\left[2 s-N t^{N-2 s}+(N-2 s) t^{N}\right]}{2 N}\left\|(-\triangle)^{\frac{s}{2}} u\right\|_{2}^{2}, \\
& \forall u \in H^{s}\left(\mathbb{R}^{N}\right), t>0 .
\end{aligned}
$$

The proof is concluded.

According to the conclusion of Lemma 3.2, we deduce the following two corollaries.

Corollary 3.3 Suppose that (G1)-(G3) hold. Then

$$
\begin{aligned}
I^{\infty}(u) \geq & I^{\infty}\left(u_{t}\right)+\frac{1-t^{N}}{N} \mathcal{P}^{\infty}(u) \\
& +\frac{2 s-N t^{N-2 s}+(N-2 s) t^{N}}{2 N}\left\|(-\triangle)^{\frac{s}{2}} u\right\|_{2}^{2}, \quad \forall u \in H^{s}\left(\mathbb{R}^{N}\right), t>0 .
\end{aligned}
$$

Corollary 3.4 Suppose that (V1), (V4), (G1)-(G3) hold. Then, for $u \in \mathcal{M}$,

$$
I(u)=\max _{t>0} I\left(u_{t}\right)
$$

Lemma 3.5 Suppose that (V1), (V3), (V4) hold. Then there exist two constants $\omega_{1}, \omega_{2}>0$ such that

$$
\begin{aligned}
\omega_{1}\|u\|^{2} \leq & (N-2 s)\left\|(-\triangle)^{\frac{s}{2}} u\right\|_{2}^{2} \\
& +\int_{\mathbb{R}^{N}}[N V(x)+\nabla V(x) \cdot x] u^{2} \mathrm{~d} x \leq \omega_{2}\|u\|^{2}, \quad \forall u \in H^{s}\left(\mathbb{R}^{N}\right) .
\end{aligned}
$$


Proof Taking $t=0$ and $t \rightarrow \infty$ in (3.2) respectively, together with (V3), we deduce that

$$
-\frac{K(s) \theta(N-2 s)}{|x|^{2 s}}+N V_{\infty} \leq N V(x)+\nabla V(x) \cdot x \leq N V_{\infty}+\frac{2 K(s) \theta s}{|x|^{2 s}} .
$$

Combining (3.4) and (3.9), one has

$$
\begin{aligned}
(N & -2 s)\left\|(-\triangle)^{\frac{s}{2}} u\right\|_{2}^{2}+\int_{\mathbb{R}^{N}}[N V(x)+\nabla V(x) \cdot x] u^{2} \mathrm{~d} x \\
& \leq[N-2(1-\theta) s]\left\|(-\triangle)^{\frac{s}{2}} u\right\|_{2}^{2}+N V_{\infty}\|u\|_{2}^{2} \\
& \leq\left[N-2(1-\theta) s+N V_{\infty}\right]\|u\|^{2}:=\omega_{2}\|u\|^{2}, \quad \forall u \in H^{s}\left(\mathbb{R}^{N}\right)
\end{aligned}
$$

and

$$
\begin{aligned}
& (N-2 s)\left\|(-\triangle)^{\frac{s}{2}} u\right\|_{2}^{2}+\int_{\mathbb{R}^{N}}[N V(x)+\nabla V(x) \cdot x] u^{2} \mathrm{~d} x \\
& \quad \geq(1-\theta)(N-2 s)\left\|(-\triangle)^{\frac{s}{2}} u\right\|_{2}^{2}+N V_{\infty}\|u\|_{2}^{2} \\
& \quad \geq \min \left\{(1-\theta)(N-2 s), N V_{\infty}\right\}\|u\|^{2}:=\omega_{1}\|u\|^{2}, \quad \forall u \in H^{s}\left(\mathbb{R}^{N}\right),
\end{aligned}
$$

which completes the proof of (3.8).

In order to prove $\mathcal{M} \neq \emptyset$, we give the following lemma on the set of $\Theta$ defined as follows:

$$
\Theta=\left\{u \in H^{s}\left(\mathbb{R}^{N}\right): \int_{\mathbb{R}^{N}}\left[\frac{1}{2} V_{\infty} u^{2}-G(u)\right] \mathrm{d} x<0\right\} .
$$

Lemma 3.6 Suppose that the conditions of Theorem 1.1 hold. Then $\Theta \neq \emptyset$ and

$$
\left\{u \in H^{s}\left(\mathbb{R}^{N}\right) \backslash\{0\}: \mathcal{P}^{\infty}(u) \leq 0 \text { or } \mathcal{P}(u) \leq 0\right\} \subset \Theta .
$$

Proof Similar to the idea of [3, Theorem 2], it follows from (G4) that $\Theta \neq \emptyset$. Then there are the following two alternatives to occur:

(1) $u \in H^{s}\left(\mathbb{R}^{N}\right) \backslash\{0\}$ and $\mathcal{P}^{\infty}(u) \leq 0$;

(2) $u \in H^{s}\left(\mathbb{R}^{N}\right) \backslash\{0\}$ and $\mathcal{P}(u) \leq 0$.

If we assume that alternative (1) happens, (1.6) implies $u \in \Theta$ clearly. When alternative (2) happens, then (1.8), (3.4), and (3.9) yield

$$
\begin{aligned}
& N \int_{\mathbb{R}^{N}}\left[\frac{1}{2} V_{\infty} u^{2}-G(u)\right] \mathrm{d} x \\
& \quad=\mathcal{P}(u)-\frac{N-2 s}{2}\left\|(-\triangle)^{\frac{s}{2}} u\right\|_{2}^{2}-\frac{N}{2} \int_{\mathbb{R}^{N}}\left[\left(V(x)-V_{\infty}\right)+\frac{\nabla V(x) \cdot x}{N}\right] u^{2} \mathrm{~d} x \\
& \quad \leq-\frac{N-2 s}{2}\left\|(-\triangle)^{\frac{s}{2}} u\right\|_{2}^{2}+\frac{K(s) \theta(N-2 s)}{2} \int_{\mathbb{R}^{N}} \frac{u^{2}}{|x|^{2 s}} \mathrm{~d} x \\
& \quad \leq-\frac{(1-\theta)(N-2 s)}{2}\left\|(-\triangle)^{\frac{s}{2}} u\right\|_{2}^{2} \\
& \quad<0
\end{aligned}
$$

thus $u \in \Theta$. 
Lemma 3.7 Suppose that the conditions of Theorem 1.1 hold. Then, for any $u \in \Theta$, there exists a unique $t_{u}>0$ such that $u_{t_{u}} \in \mathcal{M}$.

Proof For fixed $u \in \Theta$, we consider a function $\zeta(t):=I\left(u_{t}\right)$ defined on $(0, \infty)$. From (1.8) and (3.5), one has

$$
\begin{gathered}
\zeta^{\prime}(t)=0 \Leftrightarrow \frac{N-2 s}{2} t^{N-2 s}\left\|(-\triangle)^{\frac{s}{2}} u\right\|_{2}^{2}+\frac{t^{N}}{2} \int_{\mathbb{R}^{N}}[N V(t x)+\nabla V(t x) \cdot t x] u^{2} \mathrm{~d} x \\
-N t^{N} \int_{\mathbb{R}^{N}} G(u) \mathrm{d} x \\
=0 \\
\Leftrightarrow \quad \mathcal{P}\left(u_{t}\right)=0 \quad \Leftrightarrow \quad u_{t} \in \mathcal{M} .
\end{gathered}
$$

By (V1), (V3), (G1), (3.5), and the definition of $\Theta$, one can obtain that $\lim _{t \rightarrow 0} \zeta(t)=0$, $\zeta(t)>0$ for $t>0$ small and $\zeta(t)<0$ for $t$ large. Thus $\max _{t \in(0, \infty)} \zeta(t)$ is achieved at $t_{u}>0$ so that $\zeta^{\prime}\left(t_{u}\right)=0$ and $u_{t_{u}} \in \mathcal{M}$.

Now we shall show that $t_{u}$ is unique for any $u \in \Theta$. In fact, for any given $u \in \Theta$, we choose $t_{1}, t_{2}>0$ such that $u_{t_{1}}, u_{t_{2}} \in \mathcal{M}$. Then $\mathcal{P}\left(u_{t_{1}}\right)=\mathcal{P}\left(u_{t_{2}}\right)=0$. Then (3.3) yields

$$
\begin{aligned}
I\left(u_{t_{1}}\right) & \geq I\left(u_{t_{2}}\right)+\frac{t_{1}^{N}-t_{2}^{N}}{N t_{1}^{N}} \mathcal{P}\left(u_{t_{1}}\right)+\frac{(1-\theta)\left[2 s t_{1}^{N}-N t_{1}^{2 s} t_{2}^{N-2 s}+(N-2 s) t_{2}^{N}\right]}{2 N t_{1}^{N}}\left\|(-\triangle)^{\frac{s}{2}} u_{t_{1}}\right\|_{2}^{2} \\
& =I\left(u_{t_{2}}\right)+\frac{(1-\theta)\left[2 s t_{1}^{N}-N t_{1}^{2 s} t_{2}^{N-2 s}+(N-2 s) t_{2}^{N}\right]}{2 N t_{1}^{N}}\left\|(-\triangle)^{\frac{s}{2}} u_{t_{1}}\right\|_{2}^{2}
\end{aligned}
$$

and

$$
\begin{aligned}
I\left(u_{t_{2}}\right) & \geq I\left(u_{t_{1}}\right)+\frac{t_{2}^{N}-t_{1}^{N}}{N t_{2}^{N}} \mathcal{P}\left(u_{t_{2}}\right)+\frac{(1-\theta)\left[2 s t_{2}^{N}-N t_{2}^{2 s} t_{1}^{N-2 s}+(N-2 s) t_{1}^{N}\right]}{2 N t_{2}^{N}}\left\|(-\triangle)^{\frac{s}{2}} u_{t_{2}}\right\|_{2}^{2} \\
& =I\left(u_{t_{1}}\right)+\frac{(1-\theta)\left[2 s t_{2}^{N}-N t_{2}^{2 s} t_{1}^{N-2 s}+(N-2 s) t_{1}^{N}\right]}{2 N t_{2}^{N}}\left\|(-\triangle)^{\frac{s}{2}} u_{t_{2}}\right\|_{2}^{2} .
\end{aligned}
$$

It follows from (3.16) and (3.17) that $t_{1}=t_{2}$. Then $t_{u}>0$ is unique for any $u \in \Theta$.

Similarly, we have the following corollary.

Corollary 3.8 Suppose that (G1)-(G4) hold. Then, for any $u \in \Theta$, there exists a unique $t_{u}>0$ such that $u_{t_{u}} \in \mathcal{M}^{\infty}$.

Combining Corollary 3.4, Lemma 3.6 with Lemma 3.7, we get the following lemma.

Lemma 3.9 Suppose that the conditions of Theorem 1.1 hold. Then $\mathcal{M} \neq \emptyset$ and $m:=$ $\inf _{u \in \mathcal{M}} I(u)=\inf _{u \in \Theta} \max _{t>0} I\left(u_{t}\right)$.

Lemma 3.10 Suppose that (V1), (V3), (G1)-(G3) hold. If $u_{n} \rightarrow \bar{u}$ in $H^{s}\left(\mathbb{R}^{N}\right)$, then

$$
I\left(u_{n}\right)=I(\bar{u})+I\left(u_{n}-\bar{u}\right)+o(1)
$$


and

$$
\mathcal{P}\left(u_{n}\right)=\mathcal{P}(\bar{u})+\mathcal{P}\left(u_{n}-\bar{u}\right)+o(1)
$$

The proof of this lemma is standard, so we omit it here.

Lemma 3.11 Suppose that the conditions of Theorem 1.1 hold. Then

(i) there exists $\rho_{0}>0$ such that $\|u\| \geq \rho_{0}, \forall u \in \mathcal{M}$;

(ii) $m>0$.

Proof (i) Since $\mathcal{P}(u)=0, \forall u \in \mathcal{M}$, by (G1), (G2), (G3), (1.6), (3.8), and the fractional Sobolev embedding theorem, we get

$$
\begin{aligned}
\frac{\omega_{1}}{2}\|u\|^{2} & \leq \frac{N-2 s}{2}\left\|(-\triangle)^{\frac{s}{2}} u\right\|_{2}^{2}+\frac{1}{2} \int_{\mathbb{R}^{N}}[N V(x)+\nabla V(x) \cdot x] u^{2} \mathrm{~d} x \\
& =N \int_{\mathbb{R}^{N}} G(u) \mathrm{d} x \\
& \leq \frac{\omega_{1}}{4}\|u\|^{2}+C_{1}\|u\|^{2_{s}^{*}} .
\end{aligned}
$$

This implies

$$
\|u\| \geq \rho_{0}:=\left(\frac{\omega_{1}}{4 C_{1}}\right)^{\frac{N-2 s}{4 s}}, \quad \forall u \in \mathcal{M} .
$$

(ii) For $u \in H^{s}\left(\mathbb{R}^{N}\right)$, by the Sobolev inequality, we have

$$
S_{s}\|u\|_{2_{s}^{*}}^{2} \leq\left\|(-\triangle)^{\frac{s}{2}} u\right\|_{2}^{2}
$$

By (V3), there exists $R>0$ such that $V(x) \geq \frac{V_{\infty}}{2}$ for $|x| \geq R$. From (G1), (G2), and (G3), there exists $C_{2}>0$ such that

$$
|G(t)| \leq \frac{1}{4} \min \left\{\frac{S_{s}}{\omega_{N}^{2 s / N} R^{2 s}}, V_{\infty}\right\}|t|^{2}+C_{2}|t|^{2_{s}^{*}}, \quad \forall t \in \mathbb{R},
$$

where $\omega_{N}$ denotes the volume of the unit ball of $\mathbb{R}^{N}$.

For $u \in \mathcal{M}$, let $t_{u}=\left[\frac{(N-2 s) S_{s}^{\frac{2 *}{2}}}{4 N C_{2}}\right]^{\frac{1}{2 s}}\left\|(-\triangle)^{\frac{s}{2}} u\right\|_{2}^{-\frac{2}{N-2 s}}$. Combining the Hölder inequality and the fractional Sobolev inequality, we get

$$
\begin{aligned}
\int_{\left|t_{u} x\right|<R} u^{2} \mathrm{~d} x & \leq\left(\frac{\omega_{N} R^{N}}{t_{u}^{N}}\right)^{2 s / N}\left(\int_{\left|t_{u} x\right|<R} u^{2 *} \mathrm{~d} x\right)^{2 / 2_{s}^{*}} \\
& \leq\left(\frac{\omega_{N} R^{N}}{t_{u}^{N}}\right)^{2 s / N} S_{s}^{-1}\left\|(-\triangle)^{\frac{s}{2}} u\right\|_{2}^{2}
\end{aligned}
$$

By (3.3), (3.5), (3.23), (3.24), and the Sobolev inequality, one has

$$
I(u) \geq I\left(u_{t_{u}}\right)
$$




$$
\begin{aligned}
= & \frac{t_{u}^{N-2 s}}{2}\left\|(-\triangle)^{\frac{s}{2}} u\right\|_{2}^{2}+\frac{t_{u}^{N}}{2} \int_{\mathbb{R}^{N}} V\left(t_{u} x\right) u^{2} \mathrm{~d} x-t_{u}^{N} \int_{\mathbb{R}^{N}} G(u) \mathrm{d} x \\
\geq & \frac{t_{u}^{N-2 s}}{4}\left\|(-\triangle)^{\frac{s}{2}} u\right\|_{2}^{2}+\frac{S_{s}}{4 \omega_{N}^{2 s / N} R^{2 s}} t_{u}^{N} \int_{\left|t_{u} x\right|<R} u^{2} \mathrm{~d} x+\frac{V_{\infty} t_{u}^{N}}{4} \int_{\left|t_{u} x\right| \geq R} u^{2} \mathrm{~d} x \\
& -t_{u}^{N} \int_{\mathbb{R}^{N}} G(u) \mathrm{d} x \\
\geq & \frac{t_{u}^{N-2 s}}{4}\left\|(-\triangle)^{\frac{s}{2}} u\right\|_{2}^{2}+\frac{1}{4} \min \left\{\frac{S_{s}}{\omega_{N}^{2 s / N} R^{2 s}}, V_{\infty}\right\} t_{u}^{N}\|u\|_{2}^{2}-t_{u}^{N} \int_{\mathbb{R}^{N}} G(u) \mathrm{d} x \\
\geq & \frac{t_{u}^{N-2 s}}{4}\left\|(-\triangle)^{\frac{s}{2}} u\right\|_{2}^{2}-C_{2} t_{u}^{N}\|u\|_{2_{s}^{*}}^{2_{s}^{*}} \\
\geq & \frac{t_{u}^{N-2 s}}{4}\left\|(-\triangle)^{\frac{s}{2}} u\right\|_{2}^{2}-C_{2} S_{s}^{2_{s}^{*}} t_{u}^{N}\left\|(-\triangle)^{\frac{s}{2}} u\right\|_{2}^{2_{s}^{*}} \\
= & \frac{2 s S_{s}^{\frac{N}{2 s}}}{4^{N / 2 s} C_{2}^{\frac{N-2 s}{2 s}}(N-2 s)}\left(\frac{N-2 s}{N}\right)^{\frac{N}{2 s}}>0, \quad \forall u \in \mathcal{M} .
\end{aligned}
$$

Thus we conclude that $m>0$.

Next, similar to the idea of $[33,35]$, we state the following two conclusions about the limit functional $I^{\infty}$ and the critical point of $I$.

Lemma 3.12 Suppose that (G1)-(G4) hold. Then $m^{\infty}:=\inf _{u \in \mathcal{M}} I^{\infty}(u)$ is achieved.

Proof By Lemma 3.6, Corollary 3.8, and Lemma 3.11, we obtain $\mathcal{M}^{\infty} \neq \emptyset$ and $m^{\infty}>0$. Take $\left\{u_{n}\right\} \subset \mathcal{M}^{\infty}$ to be such that $I^{\infty}\left(u_{n}\right) \rightarrow m^{\infty}$. Since $\mathcal{P}^{\infty}\left(u_{n}\right)=0$, we can deduce from (3.6) with $t \rightarrow 0$ that

$$
m^{\infty}+o(1)=I^{\infty}\left(u_{n}\right) \geq \frac{(1-\theta) s}{N}\left\|(-\triangle)^{\frac{s}{2}} u_{n}\right\|_{2}^{2} .
$$

This certifies that $\left\{\left\|(-\triangle)^{\frac{s}{2}} u_{n}\right\|_{2}\right\}$ is bounded. It thus remains to prove that $\left\{\left\|u_{n}\right\|\right\}$ is also bounded. From (G1), (G2), (1.6), and the fractional Sobolev embedding inequality, we get

$$
\begin{aligned}
\min & \left\{N-2 s, N V_{\infty}\right\}\left\|u_{n}\right\|^{2} \\
& \leq(N-2 s) \int_{\mathbb{R}^{N}}\left|(-\triangle)^{\frac{s}{2}} u_{n}\right|^{2} \mathrm{~d} x+N V_{\infty} \int_{\mathbb{R}^{N}} u_{n}^{2} \mathrm{~d} x \\
& =2 N \int_{\mathbb{R}^{N}} G\left(u_{n}\right) \mathrm{d} x \\
& \leq \frac{1}{2} \min \left\{N-2 s, N V_{\infty}\right\}\left\|u_{n}\right\|^{2}+C_{3}\left\|u_{n}\right\|_{2_{s}^{*}}^{2_{s}^{*}} \\
& \leq \frac{1}{2} \min \left\{N-2 s, N V_{\infty}\right\}\left\|u_{n}\right\|^{2}+C_{3} S_{s}^{-\frac{2 s}{2}}\left\|(-\triangle)^{\frac{s}{2}} u_{n}\right\|_{2}^{2_{s}^{*}} .
\end{aligned}
$$

This shows that $\left\{u_{n}\right\}$ is bounded in $H^{s}\left(\mathbb{R}^{N}\right)$. By Lemma 2.1 , one can easily show that there exist $\delta>0$ and $\left\{y_{n}\right\} \subset \mathbb{R}^{N}$ such that

$$
\int_{B_{1}\left(y_{n}\right)}\left|u_{n}\right|^{2} \mathrm{~d} x>\frac{\delta}{2}
$$


Set $\widetilde{u}_{n}(x)=u_{n}\left(x+y_{n}\right)$. Then $\left\|\widetilde{u}_{n}\right\|=\left\|u_{n}\right\|$,

$$
\int_{B_{1}(0)}\left|\widetilde{u}_{n}\right|^{2} \mathrm{~d} x>\frac{\delta}{2}
$$

and

$$
I^{\infty}\left(\widetilde{u}_{n}\right) \rightarrow m^{\infty}, \quad \mathcal{P}^{\infty}\left(\widetilde{u}_{n}\right)=0 .
$$

Therefore, there exists $\bar{u} \in H^{s}\left(\mathbb{R}^{N}\right) \backslash\{0\}$ such that

$$
\begin{cases}\widetilde{u}_{n} \rightarrow \bar{u}, & \text { in } H^{s}\left(\mathbb{R}^{N}\right) \\ \widetilde{u}_{n} \rightarrow \bar{u}, & \text { in } L_{\mathrm{loc}}^{p}\left(\mathbb{R}^{N}\right), \forall p \in\left[1,2_{s}^{*}\right) ; \\ \widetilde{u}_{n} \rightarrow \bar{u}, & \text { a.e. on } \mathbb{R}^{N} .\end{cases}
$$

Set $\hat{u}_{n}=\widetilde{u}_{n}-\bar{u}$. It follows from (3.29) and Lemma 3.10 that

$$
I^{\infty}\left(\widetilde{u}_{n}\right)=I^{\infty}(\bar{u})+I^{\infty}\left(\hat{u}_{n}\right)+o(1)
$$

and

$$
\mathcal{P}^{\infty}\left(\widetilde{u}_{n}\right)=\mathcal{P}^{\infty}(\bar{u})+\mathcal{P}^{\infty}\left(\hat{u}_{n}\right)+o(1) .
$$

By (1.4), (1.6), (3.28), (3.30), and (3.31), we obtain

$$
\frac{s}{N}\left\|(-\triangle)^{\frac{s}{2}} \hat{u}_{n}\right\|_{2}^{2}=m^{\infty}-\frac{s}{N}\left\|(-\triangle)^{\frac{s}{2}} \bar{u}\right\|_{2}^{2}+o(1), \quad \mathcal{P}^{\infty}\left(\hat{u}_{n}\right)=-\mathcal{P}^{\infty}(\bar{u})+o(1) .
$$

If there exists a subsequence $\left\{\hat{u}_{n_{i}}\right\}$ of $\left\{\hat{u}_{n}\right\}$ such that $\hat{u}_{n_{i}}=0$, up to this subsequence, we have

$$
I^{\infty}(\bar{u})=m^{\infty}, \quad \mathcal{P}^{\infty}(\bar{u})=0,
$$

thus the conclusion of Lemma 3.12 is proved. Next, we assume that $\hat{u}_{n} \neq 0$. Moreover, we claim that $\mathcal{P}^{\infty}(\bar{u}) \leq 0$. Otherwise, if $\mathcal{P}^{\infty}(\bar{u})>0$, then (3.32) implies $\mathcal{P}^{\infty}\left(\hat{u}_{n}\right)<0$ for large $n$. Noticing that there exists $t_{n}>0$ such that $\left(\hat{u}_{n}\right)_{t_{n}} \in \mathcal{M}^{\infty}$ by Lemma 3.6 and Corollary 3.8. From (1.4), (1.6), (3.3), and (3.32), we obtain

$$
\begin{aligned}
m^{\infty}-\frac{s}{N}\left\|(-\triangle)^{\frac{s}{2}} \bar{u}\right\|_{2}^{2}+o(1) & =\frac{s}{N}\left\|(-\triangle)^{\frac{s}{2}} \hat{u}_{n}\right\|_{2}^{2} \\
& =I^{\infty}\left(\hat{u}_{n}\right)-\frac{1}{N} \mathcal{P}^{\infty}\left(\hat{u}_{n}\right) \\
& \geq I^{\infty}\left(\left(\hat{u}_{n}\right)_{t_{n}}\right)-\frac{t_{n}^{N}}{N} \mathcal{P}^{\infty}\left(\hat{u}_{n}\right) \\
& \geq m^{\infty}-\frac{t_{n}^{N}}{N} \mathcal{P}^{\infty}\left(\hat{u}_{n}\right) \geq m^{\infty},
\end{aligned}
$$

which implies $\mathcal{P}^{\infty}(\bar{u}) \leq 0$ due to $\left\|(-\triangle)^{\frac{s}{2}} \bar{u}\right\|_{2}>0$. Since $\bar{u} \neq 0$, by Lemma 3.6 and Corollary 3.8, there exists $\bar{t}>0$ such that $\bar{u}_{\bar{t}} \in \mathcal{M}^{\infty}$. From (1.4), (1.6), (3.3), (3.33), and the weak 
semicontinuity of norm, we obtain

$$
\begin{aligned}
m^{\infty} & =\lim _{n \rightarrow \infty}\left[I^{\infty}\left(\widetilde{u}_{n}\right)-\frac{1}{N} \mathcal{P}^{\infty}\left(\widetilde{u}_{n}\right)\right] \\
& =\frac{s}{N} \lim _{n \rightarrow \infty}\left\|(-\triangle)^{\frac{s}{2}} \widetilde{u}_{n}\right\|_{2}^{2} \mathrm{~d} x \\
& \geq \frac{s}{N}\left\|(-\triangle)^{\frac{s}{2}} \bar{u}\right\|_{2}^{2} \\
& =I^{\infty}(\bar{u})-\frac{1}{N} \mathcal{P}^{\infty}(\bar{u}) \\
& \geq I^{\infty}\left(\bar{u}_{\bar{t}}\right)-\frac{\bar{t}^{N}}{N} \mathcal{P}^{\infty}(\bar{u}) \\
& \geq m^{\infty}-\frac{\bar{t}^{N}}{N} \mathcal{P}^{\infty}(\bar{u}) \geq m^{\infty},
\end{aligned}
$$

thus

$$
\mathcal{P}^{\infty}(\bar{u})=0, \quad I^{\infty}(\bar{u})=m^{\infty} .
$$

Lemma 3.13 Suppose that the conditions of Theorem 1.1 hold. If $\bar{u} \in \mathcal{M}$ and $I(\bar{u})=m$, then $\bar{u}$ is a critical point of $I$.

Proof We suppose by contradiction that $I^{\prime}(\bar{u}) \neq 0$. Then there exist $\delta>0$ and $\varrho>0$ such that

$$
\|u-\bar{u}\| \leq 3 \delta \quad \Rightarrow \quad I^{\prime}(u) \geq \varrho .
$$

Firstly, we prove that

$$
\lim _{t \rightarrow 1}\left\|\bar{u}_{t}-\bar{u}\right\|=0
$$

Otherwise, there exist $\varepsilon_{0}>0$ and a sequence $\left\{t_{n}\right\}$ such that

$$
\lim _{n \rightarrow \infty} t_{n}=1, \quad\left\|\bar{u}_{t_{n}}-\bar{u}\right\|^{2} \geq \varepsilon_{0} .
$$

Since $\bar{u} \in H^{s}\left(\mathbb{R}^{N}\right)$, there exist $U \in C\left(\mathbb{R}^{N}, \mathbb{R}\right)$ and $v \in C\left(\mathbb{R}^{N}, \mathbb{R}\right)$ such that

$$
\int_{\mathbb{R}^{N}}\left|(-\Delta)^{\frac{s}{2}} \bar{u}-U\right|^{2}<\frac{\varepsilon_{0}}{20}, \quad \int_{\mathbb{R}^{N}}|\bar{u}-v|^{2}<\frac{\varepsilon_{0}}{20} .
$$

From (3.36) and (3.37), one has

$$
\begin{aligned}
\|( & (\triangle)^{\frac{s}{2}}\left(\bar{u}_{t_{n}}\right)-(-\triangle)^{\frac{s}{2}} \bar{u} \|_{2}^{2} \\
& =\int_{\mathbb{R}^{N}}\left|(-\triangle)^{\frac{s}{2}}\left(\bar{u}_{t_{n}}\right)-(-\triangle)^{\frac{s}{2}} \bar{u}\right|^{2} \mathrm{~d} x \\
& \leq 2 \int_{\mathbb{R}^{N}}\left|(-\triangle)^{\frac{s}{2}}\left(\bar{u}_{t_{n}}\right)-U\right|^{2} \mathrm{~d} x+2 \int_{\mathbb{R}^{N}}\left|(-\triangle)^{\frac{s}{2}} \bar{u}-U\right|^{2} \mathrm{~d} x
\end{aligned}
$$




$$
\begin{aligned}
& =2 \int_{\mathbb{R}^{N}}\left|(-\triangle)^{\frac{s}{2}} \bar{u}\left(\frac{x}{t_{n}}\right)-U(x)\right|^{2} \mathrm{~d} x+2 \int_{\mathbb{R}^{N}}\left|(-\triangle)^{\frac{s}{2}} \bar{u}-U\right|^{2} \mathrm{~d} x \\
& \leq 4 t_{n}^{N-2 s} \int_{\mathbb{R}^{N}}\left|(-\triangle)^{\frac{s}{2}} \bar{u}(x)-U(x)\right|^{2} \mathrm{~d} x+4\left|t_{n}^{\frac{N-2 s}{2}}-1\right|^{2} \int_{\mathbb{R}^{N}}|U|^{2} \mathrm{~d} x+\frac{\varepsilon_{0}}{10} \\
& =4\left|t_{n}^{\frac{N-2 s}{2}}-1\right|^{2} \int_{\mathbb{R}^{N}}|U|^{2} \mathrm{~d} x+\frac{\left(1+2 t_{n}^{N-2 s}\right) \varepsilon_{0}}{10} \\
& =\frac{\varepsilon_{0}}{5}+o(1)
\end{aligned}
$$

and

$$
\begin{aligned}
\left\|\bar{u}_{t_{n}}-\bar{u}\right\|_{2}^{2} & =\int_{\mathbb{R}^{N}}\left|\bar{u}_{t_{n}}-\bar{u}\right|^{2} \mathrm{~d} x \\
& \leq 2 \int_{\mathbb{R}^{N}}\left|\bar{u}_{t_{n}}-v\right|^{2} \mathrm{~d} x+2 \int_{\mathbb{R}^{N}}|\bar{u}-v|^{2} \mathrm{~d} x \\
& \leq 4 \int_{\mathbb{R}^{N}}\left|v\left(t_{n}^{-1} x\right)-v(x)\right|^{2} \mathrm{~d} x+\frac{\left(1+2 t_{n}^{N}\right) \varepsilon_{0}}{10} \\
& =\frac{3}{10} \varepsilon_{0}+o(1) .
\end{aligned}
$$

It follows from (3.38) and (3.39) that

$$
\left\|\bar{u}_{t_{n}}-\bar{u}\right\|^{2}=\left\|(-\triangle)^{\frac{s}{2}}\left(\bar{u}_{t_{n}}\right)-(-\triangle)^{\frac{s}{2}} \bar{u}\right\|_{2}^{2}+\left\|\bar{u}_{t_{n}}-\bar{u}\right\|_{2}^{2} \leq \frac{\varepsilon_{0}}{2}+o(1)
$$

(3.40) contradicts (3.36). Then (3.35) holds. It is thus clear that there exists $\delta_{1} \in(0,1 / 4)$ such that

$$
|t-1|<\delta_{1} \quad \Rightarrow \quad\left\|\bar{u}_{t}-\bar{u}\right\|<\delta .
$$

Thanks to Lemma 3.2, we have

$$
\begin{aligned}
I\left(\bar{u}_{t}\right) & \leq I(\bar{u})-\frac{(1-\theta)\left[2 s-N t^{N-2 s}+(N-2 s) t^{N}\right]}{2 N}\left\|(-\triangle)^{\frac{s}{2}} \bar{u}\right\|_{2}^{2} \\
& =m-\frac{(1-\theta) h(t)}{2 N}\left\|(-\triangle)^{\frac{s}{2}} \bar{u}\right\|_{2}^{2}, \quad \forall t>0 .
\end{aligned}
$$

It follows from (1.8), (3.4), and (3.9) that there exist $T_{1} \in(0,1)$ and $T_{2} \in(1, \infty)$ such that

$$
\mathcal{P}\left(\bar{u}_{T_{1}}\right)>0, \quad \mathcal{P}\left(\bar{u}_{T_{2}}\right)<0 .
$$

Let $\varepsilon:=\min \left\{(1-\theta) h\left(T_{1}\right)\left\|(-\triangle)^{\frac{s}{2}} \bar{u}\right\|_{2}^{2} /(6 N),(1-\theta) h\left(T_{2}\right)\left\|(-\triangle)^{\frac{s}{2}} \bar{u}\right\|_{2}^{2} /(6 N), 1, \varrho \delta / 8\right\}$, and $S:=$ $B(\bar{u}, \delta)$. Then [27, Theorem 5.3.7] yields a deformation $\eta \in C\left([0,1] \times H^{s}\left(\mathbb{R}^{N}\right), H^{s}\left(\mathbb{R}^{N}\right)\right)$ such that

(i) $\eta(1, u)=u$ if $I(u)<m-2 \varepsilon$ or $I(u)>m+2 \varepsilon$;

(ii) $\eta\left(1, I^{m+\varepsilon} \cap B(\bar{u}, \delta)\right) \subset I^{m-\varepsilon}$;

(iii) $I(\eta(1, u)) \leq I(u), \forall u \in H^{s}\left(\mathbb{R}^{N}\right)$;

(iv) $\eta(1, u)$ is a homeomorphism of $H^{s}\left(\mathbb{R}^{N}\right)$. 
By Corollary 3.4, $I\left(\bar{u}_{t}\right) \leq I(\bar{u})=m$ for $t>0$, then it follows from (3.41) and (ii) that

$$
I\left(\eta\left(1, \bar{u}_{t}\right)\right) \leq m-\varepsilon, \quad \forall t>0,|t-1|<\delta_{1} .
$$

On the other hand, by (iii) and (3.42), one has

$$
\begin{aligned}
I\left(\eta\left(1, \bar{u}_{t}\right)\right) & \leq I\left(\bar{u}_{t}\right) \\
& \leq m-\frac{(1-\theta)\left[2 s-N t^{N-2 s}+(N-2 s) t^{N}\right]}{2 N}\left\|(-\triangle)^{\frac{s}{2}} \bar{u}\right\|_{2}^{2} \\
& \leq m-\frac{(1-\theta) \delta_{2}}{2 N}\left\|(-\triangle)^{\frac{s}{2}} \bar{u}\right\|_{2}^{2}, \quad \forall t>0,|t-1| \geq \delta_{1},
\end{aligned}
$$

where $\delta_{2}:=\min \left\{h\left(1-\delta_{1}\right), h\left(1+\delta_{1}\right)\right\}>0$. Combining (3.44) with (3.45), we have

$$
\max _{t \in\left[T_{1}, T_{2}\right]} I\left(\eta\left(1, \bar{u}_{t}\right)\right)<m
$$

Next we shall prove that $\eta\left(1, \bar{u}_{t}\right) \cap \mathcal{M} \neq \emptyset$, contradicting the definition of $m$. Define

$$
\psi_{0}(t):=\mathcal{P}\left(\eta\left(1, \bar{u}_{t}\right)\right), \quad \forall t>0
$$

According to (i), (3.42), and (3.43), since

$$
\psi_{0}\left(T_{1}\right)=\mathcal{P}\left(\eta\left(1, \bar{u}_{T_{1}}\right)\right)=\mathcal{P}\left(\bar{u}_{T_{1}}\right)>0, \quad \psi_{0}\left(T_{2}\right)=\mathcal{P}\left(\eta\left(1, \bar{u}_{T_{2}}\right)=\mathcal{P}\left(\bar{u}_{T_{2}}\right)<0,\right.
$$

the existence of $t \in\left(T_{1}, T_{2}\right)$ with $\psi_{0}(t)=0$, i.e., $\eta\left(1, \bar{u}_{t}\right) \cap \mathcal{M} \neq \emptyset$ follows from the intermediate value theorem.

So far, we could draw a conclusion on the existence of a ground state solution of Pohozaev type to the "limit problem" of Problem (1.1), that is, Theorem 1.2. We also have the following corollary of Theorem 1.2.

Corollary 3.14 Let $\tilde{g}(t)=0$ for $t<0$ and $\tilde{g}(t)=g(t)$ for $t \geq 0$. Set $\tilde{g}$ to take place of $g$ in (1.3) and assume (G1)-(G4) hold, then Problem (1.3) has a positive ground state solution $\bar{u} \in H^{s}\left(\mathbb{R}^{N}\right) \backslash\{0\}$ such that $I^{\infty}(\bar{u})=\inf _{\mathcal{M}^{\infty}} I^{\infty}>0$.

Then our task is to give the proof of Theorem 1.1. Next, we give the following comparison between $m$ and $m^{\infty}$.

Lemma 3.15 Suppose that (V1), (V3), (V4), and (G1)-(G3) hold. Then $m^{\infty} \geq m$.

Proof By virtue of Theorem 1.2, $I^{\infty}$ has a minimizer $u^{\infty} \neq 0$ on $\mathcal{M}^{\infty}$, i.e.,

$$
u^{\infty} \in \mathcal{M}^{\infty} \text { and } m^{\infty}=I^{\infty}\left(u^{\infty}\right)
$$

By Lemma 3.6, there exists $t_{0}>0$ such that $\left(u^{\infty}\right)_{t_{0}} \in \mathcal{M}$. Thus, (V3), (1.2), (1.4), (3.6), (3.47) yield

$$
m^{\infty}=I^{\infty}\left(u^{\infty}\right) \geq I^{\infty}\left(\left(u^{\infty}\right)_{t_{0}}\right)>I\left(\left(u^{\infty}\right)_{t_{0}}\right) \geq m
$$


Lemma 3.16 Suppose that the conditions of Theorem 1.1 hold. Then $m$ is achieved.

The idea of the proof of Lemma 3.16 is the same as that of Lemma 3.12, we omit the detailed proof here.

Proof of Theorem 1.1 Owing to Lemmas 3.9, 3.13, and 3.16, there exists $\bar{u} \in \mathcal{M}$ such that

$$
I(\bar{u})=m=\inf _{u \in \Theta} \max _{t>0} I\left(u_{t}\right), \quad I^{\prime}(\bar{u})=0 .
$$

This certifies that $\bar{u}$ is a ground state solution of Pohožaev type for (1.1).

\section{Existence of the least energy solution for (1.1)}

The purpose of this section is to give the proof of Theorem 1.3. We begin with introducing the following Pohožaev identity.

Lemma 4.1 ([30]) Assume that (V1), (V3), (G1)-(G3) hold. Let u be a critical point of $I_{\lambda}$ in $H^{s}\left(\mathbb{R}^{N}\right)$, then we have the following Pohozaaev type identity:

$$
\begin{aligned}
\mathcal{P}_{\lambda}(u):= & \frac{N-2 s}{2}\left\|(-\triangle)^{\frac{s}{2}} u\right\|_{2}^{2}+\frac{1}{2} \int_{\mathbb{R}^{N}}[N V(x)+\nabla V(x) \cdot x] u^{2} \mathrm{~d} x \\
& -N \lambda \int_{\mathbb{R}^{N}} F(u) \mathrm{d} x=0 .
\end{aligned}
$$

In view of Corollary 3.3, we have the following lemma.

Lemma 4.2 Suppose that (G1)-(G3) hold. Then

$$
\begin{gathered}
I_{\lambda}^{\infty}(u) \geq I_{\lambda}^{\infty}\left(u_{t}\right)+\frac{1-t^{N}}{N} \mathcal{P}_{\lambda}^{\infty}(u)+\frac{2 s-N t^{N-2 s}+(N-2 s) t^{N}}{2 N}\left\|(-\triangle)^{\frac{s}{2}} u\right\|_{2}^{2}, \\
\forall u \in H^{s}\left(\mathbb{R}^{N}\right), t>0, \lambda \geq 0 .
\end{gathered}
$$

By virtue of Theorem $1.2, I_{1}^{\infty}=I^{\infty}$ has a minimizer $u_{1}^{\infty} \neq 0$ on $\mathcal{M}_{1}^{\infty}=\mathcal{M}^{\infty}$, i.e.,

$$
u_{1}^{\infty} \in \mathcal{M}_{1}^{\infty}, \quad\left(I_{1}^{\infty}\right)^{\prime}\left(u_{1}^{\infty}\right)=0 \quad \text { and } \quad m_{1}^{\infty}=I_{1}^{\infty}\left(u_{1}^{\infty}\right)
$$

where

$$
m_{\lambda}^{\infty}:=\inf _{u \in \mathcal{M}_{\lambda}^{\infty}} I_{\lambda}^{\infty}(u)
$$

We use the ingenious assumptions on $V$ borrowed from [35], that is, for $V \in C\left(\mathbb{R}^{N}, \mathbb{R}\right)$ and $V(x) \leq V_{\infty}$ but $V(x) \not \equiv V_{\infty}$, then there exist $\bar{x} \in \mathbb{R}^{N}$ and $\bar{r}>0$ such that

$$
V_{\infty}-V(x)>0, \quad\left|u_{1}^{\infty}(x)\right|>0 \quad \text { a.e. }|x-\bar{x}| \leq \bar{r} .
$$

Next, similar to the local counterpart, we conclude $I_{\lambda}$ with $\lambda \in[0.5,1]$ has the following features. 
Lemma 4.3 Assume that (V1), (V3), and (G1)-(G4) hold. Then

(i) there exists $T>0$ independent of $\lambda$ such that $I_{\lambda}\left(\left(u_{1}^{\infty}\right)_{T}\right)<0$ for all $\lambda \in[0.5,1]$;

(ii) there exists $\kappa_{0}>0$ independent of $\lambda$ such that, for all $\lambda \in[0.5,1]$,

$$
c_{\lambda}:=\inf _{\gamma \in \Upsilon} \max _{\zeta \in[0,1]} I_{\lambda}(\gamma(\varsigma)) \geq \kappa_{0}>\max \left\{I_{\lambda}(0), I_{\lambda}\left(\left(u_{1}^{\infty}\right)_{T}\right)\right\}
$$

where

$$
\Upsilon=\left\{\gamma \in C\left([0,1], H^{s}\left(\mathbb{R}^{N}\right)\right): \gamma(0)=0, \gamma(1)=\left(u_{1}^{\infty}\right)_{T}\right\}
$$

(iii) $c_{\lambda}$ is bounded for $\lambda \in[0.5,1]$;

(iv) $m_{\lambda}^{\infty}$ is nonincreasing on $\lambda \in[0.5,1]$;

(v) $\lim \sup _{\lambda \rightarrow \lambda_{0}} c_{\lambda} \leq c_{\lambda_{0}}$ for $\lambda_{0} \in(0.5,1]$.

By the similar argument as that in Lemma 4.5 in [35], we obtain the following crucial inequality in order to recover the compactness.

Lemma 4.4 Assume that (V1), (V3), and (G1)-(G4) hold. Then there exists $\bar{\lambda} \in[0.5,1)$ such that $c_{\lambda}<m_{\lambda}^{\infty}$ for $\lambda \in[\bar{\lambda}, 1]$.

Proof It is clear that, for any $\lambda \in[0.5,1]$, we can choose $t_{\lambda} \in(0, T)$ such that $I_{\lambda}\left(\left(u_{1}^{\infty}\right)_{t_{\lambda}}\right)=$ $\max _{t \in[0, T]} I_{\lambda}\left(\left(u_{1}^{\infty}\right)_{t}\right)$. Define

$$
\gamma_{0}(t)= \begin{cases}\left(u_{1}^{\infty}\right)_{t T}, & \text { for } t>0, \\ 0, & \text { for } t=0 .\end{cases}
$$

Then $\gamma_{0} \in \Upsilon$ defined by Lemma 4.3(ii), i.e., $\gamma_{0}(0)=0, \gamma_{0}(1)=\left(u_{1}^{\infty}\right)_{T}$. Moreover,

$$
I_{\lambda}\left(\left(u_{1}^{\infty}\right)_{t_{\lambda}}\right)=\max _{t \in[0,1]} I_{\lambda}\left(\gamma_{0}(t)\right) \geq c_{\lambda}
$$

Let

$$
\zeta_{0}:=\min \{3 \bar{r} / 8(1+|\bar{x}|), 1 / 4\}
$$

Then it follows from (4.4) and (4.6) that

$$
|x-\bar{x}| \leq \frac{\bar{r}}{2} \quad \text { and } \quad \tau \in\left[1-\zeta_{0}, 1+\zeta_{0}\right] \quad \Rightarrow \quad|\tau x-\bar{x}| \leq \bar{r}
$$

Let

$$
\begin{gathered}
\bar{\lambda}:=\max \left\{\frac{1}{2}, 1-\frac{\left(1-\zeta_{0}\right)^{N} \min _{\tau \in\left[1-\zeta_{0}, 1+\zeta_{0}\right]} \int_{\mathbb{R}^{N}}\left[V_{\infty}-V(\tau x)\right]\left|u_{1}^{\infty}\right|^{2} \mathrm{~d} x}{T^{N} \int_{\mathbb{R}^{N}} G\left(u_{1}^{\infty}\right) \mathrm{d} x},\right. \\
\left.1-\frac{\min \left\{h\left(1-\zeta_{0}\right), h\left(1+\zeta_{0}\right)\right\}\left\|(-\triangle)^{\frac{s}{2}} u_{1}^{\infty}\right\|_{2}^{2}}{N T^{N} \int_{\mathbb{R}^{N}} G\left(u_{1}^{\infty}\right) \mathrm{d} x}\right\} .
\end{gathered}
$$

Then it follows from (3.1), (4.4), and (4.7) that $1 / 2 \leq \bar{\lambda}<1$. Additionally, since $\mathcal{P}^{\infty}\left(u_{1}^{\infty}\right)=0$, then $\int_{\mathbb{R}^{N}} G\left(u_{1}^{\infty}\right) \mathrm{d} x>0$. Next, we have two cases to distinguish: 
Case (i). $t_{\lambda} \in\left[1-\zeta_{0}, 1+\zeta_{0}\right]$. From(1.10), (1.11), (4.2)-(4.5), (4.7), (4.8), and Lemma 4.3(iv), we have

$$
\begin{aligned}
m_{\lambda}^{\infty} \geq & m_{1}^{\infty}=I_{1}^{\infty}\left(u_{1}^{\infty}\right) \geq I_{1}^{\infty}\left(\left(u_{1}^{\infty}\right)_{t_{\lambda}}\right) \\
= & I_{\lambda}\left(\left(u_{1}^{\infty}\right)_{t_{\lambda}}\right)-\frac{(1-\lambda) t_{\lambda}^{N}}{2} \int_{\mathbb{R}^{N}} G\left(u_{1}^{\infty}\right) \mathrm{d} x+\frac{t_{\lambda}^{N}}{2} \int_{\mathbb{R}^{N}}\left[V_{\infty}-V\left(t_{\lambda} x\right)\right]\left|u_{1}^{\infty}\right|^{2} \mathrm{~d} x \\
> & c_{\lambda}-\frac{(1-\lambda) T^{N}}{2} \int_{\mathbb{R}^{N}} G\left(u_{1}^{\infty}\right) \mathrm{d} x \\
& \quad+\frac{\left(1-\zeta_{0}\right)^{N}}{2} \min _{\tau \in\left[1-\zeta_{0}, 1+\zeta_{0}\right]} \int_{\mathbb{R}^{N}}\left[V_{\infty}-V(\tau x)\right]\left|u_{1}^{\infty}\right|^{2} \mathrm{~d} x \\
\geq & c_{\lambda}, \quad \forall \lambda \in[\bar{\lambda}, 1] .
\end{aligned}
$$

Case (ii). $t_{\lambda} \in\left(0,1-\zeta_{0}\right) \cup\left(1+\zeta_{0}, T\right]$. From (1.10), (1.11), (3.1), (4.2), (4.4), (4.5), (4.8), and Lemma 4.3(iv),

$$
\begin{aligned}
m_{\lambda}^{\infty} \geq & m_{1}^{\infty}=I_{1}^{\infty}\left(u_{1}^{\infty}\right) \geq I_{1}^{\infty}\left(\left(u_{1}^{\infty}\right)_{t_{\lambda}}\right)+\frac{h\left(t_{\lambda}\right)\left\|(-\triangle)^{\frac{s}{2}} u_{1}^{\infty}\right\|_{2}^{2}}{2 N} \\
= & I_{\lambda}\left(\left(u_{1}^{\infty}\right)_{t_{\lambda}}\right)-\frac{(1-\lambda) t_{\lambda}^{N}}{2} \int_{\mathbb{R}^{N}} G\left(u_{1}^{\infty}\right) \mathrm{d} x \\
& \quad+\frac{t_{\lambda}^{N}}{2} \int_{\mathbb{R}^{N}}\left[V_{\infty}-V\left(t_{\lambda} x\right)\right]\left|u_{1}^{\infty}\right|^{2} \mathrm{~d} x+\frac{h\left(t_{\lambda}\right)\left\|(-\triangle)^{\frac{s}{2}} u_{1}^{\infty}\right\|_{2}^{2}}{2 N} \\
> & c_{\lambda}-\frac{(1-\lambda) T^{N}}{2} \int_{\mathbb{R}^{N}} G\left(u_{1}^{\infty}\right) \mathrm{d} x+\frac{\min \left\{h\left(1-\zeta_{0}\right), h\left(1+\zeta_{0}\right)\right\}\left\|(-\triangle)^{\frac{s}{2}} u_{1}^{\infty}\right\|_{2}^{2}}{2 N} \\
\geq & c_{\lambda}, \quad \forall \lambda \in(\bar{\lambda}, 1] .
\end{aligned}
$$

Combining both the above cases, we therefore have $c_{\lambda}<m_{\lambda}^{\infty}$ for $\lambda \in(\bar{\lambda}, 1]$.

In what follows we use profile decomposition to obtain the compactness for any bounded (PS) sequence of the perturbed functional, which is also called compactness splitting lemma in [25].

Lemma 4.5 ([25]) Assume that (V1), (V3), and (G1)-(G4) hold. Let $\left\{u_{n}\right\}$ be a bounded (PS) sequence for $I_{\lambda}$ with $\lambda \in[0.5,1]$. Then there exist a subsequence of $\left\{u_{n}\right\}$, still denoted by $\left\{u_{n}\right\}$, and $u_{0} \in H^{s}\left(\mathbb{R}^{N}\right)$, an integer $l \in \mathbb{N} \cup\{0\}$, a sequence $\left\{y_{n}^{k}\right\}$, and $w^{k} \in H^{s}\left(\mathbb{R}^{N}\right)$ for $1 \leq k \leq l$, such that

(i) $u_{n} \rightarrow u_{0}$ with $I_{\lambda}^{\prime}\left(u_{0}\right)=0$;

(ii) $w^{k} \neq 0$ and $\left(I_{\lambda}^{\infty}\right)^{\prime}\left(w^{k}\right)=0$ for $1 \leq k \leq l$;

(iii) $\left\|u_{n}-u_{0}-\sum_{k=1}^{l} w^{k}\left(\cdot+y_{n}^{k}\right)\right\| \rightarrow 0$, as $n \rightarrow \infty$;

(iv) $I_{\lambda}\left(u_{n}\right) \rightarrow I_{\lambda}\left(u_{0}\right)+\sum_{i=1}^{l} I_{\lambda}^{\infty}\left(w^{i}\right)$;

where we agree that in the case $l=0$ the above holds without $w^{k}$.

Lemma 4.6 Assume that (V1), (V3), (V5), and (G1)-(G4) hold. Then, for almost every $\lambda \in(\bar{\lambda}, 1]$, there exists $u_{\lambda} \in H^{s}\left(\mathbb{R}^{N}\right) \backslash\{0\}$ such that

$$
I_{\lambda}^{\prime}\left(u_{\lambda}\right)=0, \quad I_{\lambda}\left(u_{\lambda}\right)=c_{\lambda} .
$$


Proof Under the assumptions of (V1), (V3), and (G1)-(G4), Lemma 4.4 implies that $I_{\lambda}(u)$ satisfies the assumptions of Proposition 2.2 with $X=H^{s}\left(\mathbb{R}^{N}\right), \Lambda=[\bar{\lambda}, 1]$, and $\Phi_{\lambda}=I_{\lambda}$. So, for almost every $\lambda \in(\bar{\lambda}, 1]$, there exists a bounded sequence $\left\{u_{n}(\lambda)\right\} \subset H^{s}\left(\mathbb{R}^{N}\right)$ (for simplicity, we denote the sequence by $\left\{u_{n}\right\}$ instead of $\left.\left\{u_{n}(\lambda)\right\}\right)$ such that

$$
I_{\lambda}\left(u_{n}\right) \rightarrow c_{\lambda}>0, \quad\left\|I_{\lambda}^{\prime}\left(u_{n}\right)\right\| \rightarrow 0
$$

From Lemmas 4.1 and 4.5, there exists a subsequence of $\left\{u_{n}\right\}$, still denoted by $\left\{u_{n}\right\}$, and $u_{\lambda} \in H^{s}\left(\mathbb{R}^{N}\right)$, an integer $l \in \mathbb{N} \cup\{0\}$, a sequence $w^{1}, \ldots, w^{l} \in H^{s}\left(\mathbb{R}^{N}\right) \backslash\{0\}$ such that

$$
\begin{aligned}
& u_{n} \rightarrow u_{\lambda} \quad \text { in } H^{s}\left(\mathbb{R}^{N}\right), \quad\left(I_{\lambda}\right)^{\prime}\left(u_{\lambda}\right)=0, \\
& \left(I_{\lambda}^{\infty}\right)^{\prime}\left(w^{k}\right)=0, \quad\left(I_{\lambda}^{\infty}\right)^{\prime}\left(w^{k}\right) \geq m_{\lambda}^{\infty}, \quad 1 \leq k \leq l,
\end{aligned}
$$

and

$$
c_{\lambda}=I_{\lambda}\left(u_{\lambda}\right)+\sum_{i=1}^{l} I_{\lambda}^{\infty}\left(w^{i}\right)
$$

Note that $\left(I_{\lambda}\right)^{\prime}\left(u_{\lambda}\right)=0$, then it follows from Lemma 4.1 that

$$
\begin{aligned}
\mathcal{P}_{\lambda}\left(u_{\lambda}\right):= & \frac{N-2 s}{2}\left\|(-\triangle)^{\frac{s}{2}} u_{\lambda}\right\|_{2}^{2}+\frac{1}{2} \int_{\mathbb{R}^{N}}[N V(x)+\nabla V(x) \cdot x] u_{\lambda}^{2} \mathrm{~d} x \\
& -N \lambda \int_{\mathbb{R}^{N}} G\left(u_{\lambda}\right) \mathrm{d} x \\
= & 0 .
\end{aligned}
$$

Since $\left\|u_{n}\right\| \nrightarrow 0$, then (4.11) and (4.12) yield that if $u_{\lambda}=0$ then $l \geq 1$ and

$$
c_{\lambda}=I_{\lambda}\left(u_{\lambda}\right)+\sum_{i=1}^{l} I_{\lambda}^{\infty}\left(w^{i}\right) \geq m_{\lambda}^{\infty},
$$

which contradicts Lemma 4.4. Thus $u_{\lambda} \neq 0$. By (1.10), (3.4), (4.14), and (V5), one has

$$
\begin{aligned}
I_{\lambda}\left(u_{\lambda}\right) & =I_{\lambda}\left(u_{\lambda}\right)-\frac{1}{N} \mathcal{P}_{\lambda}\left(u_{\lambda}\right) \\
& =\frac{s}{N}\left\|(-\triangle)^{\frac{s}{2}} u_{\lambda}\right\|_{2}^{2}-\frac{1}{2 N} \int_{\mathbb{R}^{N}} \nabla V(x) \cdot x u_{\lambda}^{2} \mathrm{~d} x \\
& \geq \frac{s-\hat{\theta}}{N}\left\|(-\triangle)^{\frac{s}{2}} u_{\lambda}\right\|_{2}^{2}>0 .
\end{aligned}
$$

Combining (4.13) and (4.15), we obtain

$$
c_{\lambda}=I_{\lambda}\left(u_{\lambda}\right)+\sum_{i=1}^{l} I_{\lambda}^{\infty}\left(w^{i}\right)>\operatorname{lm}_{\lambda}^{\infty} .
$$

From Lemma 4.4, we have $c_{\lambda}<m_{\lambda}^{\infty}$ for $\lambda \in(\bar{\lambda}, 1]$, which works jointly with (4.16), implies that $l=0$ and $I_{\lambda}\left(u_{\lambda}\right)=c_{\lambda}$. 
Lemma 4.7 Suppose that (V1), (V3), (V5), and (G1)-(G4) hold. Then there exists $\bar{u} \in$ $H^{s}\left(\mathbb{R}^{N}\right) \backslash\{0\}$ such that

$$
I^{\prime}(\bar{u})=0, \quad 0<I(\bar{u})<c_{1} .
$$

Proof By virtue of Lemma 4.6, there exist two sequences $\left\{\lambda_{n}\right\} \subset[\bar{\lambda}, 1]$ and $u_{\lambda_{n}} \subset H^{s}\left(\mathbb{R}^{N}\right) \backslash$ $\{0\}$ denoted by $\left\{u_{n}\right\}$ such that

$$
\lambda_{n} \rightarrow 1, \quad c_{\lambda_{n}} \rightarrow c_{*}, \quad I_{\lambda_{n}}^{\prime}\left(u_{n}\right)=0, \quad 0<I_{\lambda_{n}}\left(u_{n}\right) \leq c_{\lambda_{n}} .
$$

Then it follows from Lemma 4.1 that

$$
\begin{aligned}
\mathcal{P}_{\lambda_{n}}\left(u_{n}\right) & :=\frac{N-2 s}{2}\left\|(-\triangle)^{\frac{s}{2}} u_{\lambda}\right\|_{2}^{2}+\frac{1}{2} \int_{\mathbb{R}^{N}}[N V(x)+\nabla V(x) \cdot x] u_{n}^{2} \mathrm{~d} x-N \lambda \int_{\mathbb{R}^{N}} G\left(u_{n}\right) \mathrm{d} x \\
& =0 .
\end{aligned}
$$

By (V5), (1.10), (3.4), (4.18), and (4.19) and Lemma 4.3(iii), we have

$$
\begin{aligned}
C_{5} & \geq c_{\lambda_{n}}=I_{\lambda_{n}}\left(u_{n}\right)-\frac{1}{N} \mathcal{P}_{\lambda_{n}}\left(u_{n}\right) \\
& =\frac{s}{N}\left\|(-\triangle)^{\frac{s}{2}} u_{n}\right\|_{2}^{2}-\frac{1}{2 N} \int_{\mathbb{R}^{N}} \nabla V(x) \cdot x u_{n}^{2} \mathrm{~d} x \\
& \geq \frac{s-\hat{\theta}}{N}\left\|(-\triangle)^{\frac{s}{2}} u_{n}\right\|_{2}^{2} .
\end{aligned}
$$

We therefore conclude that $\left\{\left\|(-\triangle)^{\frac{s}{2}} u_{n}\right\|_{2}\right\}$ is bounded. Next, it thus remains to show that $\left\{u_{n}\right\}$ is bounded in $H^{s}\left(\mathbb{R}^{N}\right)$. From (V1)-(V3), it is easy to certify that there exists a constant $\omega_{3}>0$ such that

$$
\int_{\mathbb{R}^{N}}\left[\left|(-\triangle)^{\frac{s}{2}} u\right|^{2}+V(x) u^{2}\right] \mathrm{d} x \geq \omega_{3}\|u\|^{2}, \quad \forall u \in H^{s}\left(\mathbb{R}^{N}\right) .
$$

By (G1)-(G3), (1.10), (4.18), (4.20), (4.21), Lemma 4.3(iii), and the fractional Sobolev embedding inequality, we have

$$
\begin{aligned}
\omega_{3}\left\|u_{n}\right\|^{2} & \leq \int_{\mathbb{R}^{N}}\left[\left|(-\triangle)^{\frac{s}{2}} u_{n}\right|^{2}+V(x) u_{n}^{2}\right] \mathrm{d} x \\
& \leq 2 c_{\lambda_{n}}+2 \lambda_{n} \int_{\mathbb{R}^{N}} G\left(u_{n}\right) \mathrm{d} x \\
& \leq 2 C_{5}+\frac{\omega_{3}}{2}\left\|u_{n}\right\|^{2}+C_{6}\left\|u_{n}\right\|_{2_{s}^{*}}^{2^{*}} \\
& \leq 2 C_{5}+\frac{\omega_{3}}{2}\left\|u_{n}\right\|^{2}+C_{6} S_{s}^{-\frac{2_{s}^{*}}{2}}\left\|(-\triangle)^{\frac{s}{2}} u_{n}\right\|_{2}^{2_{s}^{*}} .
\end{aligned}
$$

This shows that $\left\{u_{n}\right\}$ is bounded in $H^{s}\left(\mathbb{R}^{N}\right)$. From Lemma 4.3(v), then $\lim _{n \rightarrow \infty} c_{\lambda_{n}}=c_{*} \leq c_{1}$ hold. Consequently, by (1.2), (1.10), and (4.18), it follows that $I\left(u_{n}\right) \rightarrow c_{*}$ and $I^{\prime}\left(u_{n}\right) \rightarrow 0$. Thus $\left\{u_{n}\right\}$ satisfy (4.10) with $c_{\lambda}=c_{*}$. The arguments of Lemma 4.6 show that there exists $\bar{u} \in H^{s}\left(\mathbb{R}^{N}\right) \backslash\{0\}$ such that (4.17) holds. 
Proof of Theorem 1.3 Let

$$
\Sigma:=\left\{u \in H^{s}\left(\mathbb{R}^{N}\right) \backslash\{0\}: I^{\prime}(u)=0\right\}, \quad \hat{m}=\inf _{u \in \Sigma} I(u) .
$$

It follows from Lemma 4.7 that $\Sigma \neq \emptyset$ and $\hat{m} \leq c_{1}$. For any $u \in \Sigma$, Lemma 4.1 yields $\mathcal{P}(u)=$ $\mathcal{P}_{1}(u)=0$. Therefore, it follows from (4.15) that $I(u)=I_{1}(u)>0$, thus $\hat{m} \geq 0$. Set $\left\{u_{n}\right\} \subset \Sigma$ such that

$$
I^{\prime}\left(u_{n}\right)=0, \quad I\left(u_{n}\right) \rightarrow \hat{m} .
$$

By Lemma 4.4, we have $\hat{m} \leq c_{1}<m_{1}^{\infty}$. Through a similar argument as in the proof of Lemma 4.6, we can certify that there exists $\bar{u} \in H^{s}\left(\mathbb{R}^{N}\right) \backslash\{0\}$ such that

$$
I^{\prime}(\bar{u})=0, \quad I(\bar{u})=\hat{m} .
$$

This shows that $\bar{u}$ is a nontrivial least energy solution of (1.1).

\section{Acknowledgements}

The authors would like to thank the referees for their useful suggestions.

\section{Funding}

The authors are supported financially by the National Natural Science Foundation of China (No: 11501190) and Hunan provincial Natural Science Foundation (No: 2019JJ50146)

\section{Availability of data and materials}

Not applicable.

\section{Competing interests}

The authors declare that they have no competing interests.

\section{Authors' contributions}

The research was carried out in collaboration. All authors read and assured the final manuscript.

\section{Author details}

${ }^{1}$ School of Mathematics and Computing Sciences, Hunan University of Science and Technology, Xiangtan, P.R. China.

${ }^{2}$ School of Mathematics and Statistics, Central South University, Changsha, P.R. China.

\section{Publisher's Note}

Springer Nature remains neutral with regard to jurisdictional claims in published maps and institutional affiliations.

Received: 1 July 2019 Accepted: 5 September 2019 Published online: 14 September 2019

\section{References}

1. Azzollini, A., Pomponio, A.: On the Schrödinger equation in $\mathbb{R}^{N}$ under the effect of a general nonlinear term. Indiana Univ. Math. J. 58(3), 1361-1378 (2009)

2. Beckner, W.: Pitt's inequality with sharp convolution estimates. Proc. Am. Math. Soc. 136(5), 1871-1885 (2008)

3. Berestycki, H., Lions, P.L.: Nonlinear scalar field equations I. Existence of a ground state. Arch. Ration. Mech. Anal. 82, 313-346 (1983)

4. Bogdan, K., Dyda, B.: The best constant in a fractional Hardy inequality. Math. Nachr. 284, 629-638 (2011)

5. Caffarelli, L., Silvestre, L.: An extension problem related to the fractional Laplacian. Commun. Partial Differ. Equ. 32, 1245-1260 (2007)

6. Chang, X.J.: Ground state of asymptotically linear fractional Schrödinger equations. J. Math. Phys. 54, 061504 (2013)

7. Chang, X.J., Wang, Z.Q.: Ground state of scalar field equations involving a fractional Laplacian with general nonlinearity. Nonlinearity 26(2), 479-494 (2013)

8. Chen, S.T., Shi, J.P., Tang, X.H.: Ground state solutions of Nehari-Pohozaev type for the planar Schrödinger-Poisson system with general nonlinearity. Discrete Contin. Dyn. Syst., Ser. A 39, 5867-5889 (2019)

9. Chen, S.T., Tang, X.H.: Improved results for Klein-Gordon-Maxwell systems with general nonlinearity. Discrete Contin. Dyn. Syst., Ser. A 38, 2333-2348 (2018)

10. Chen, S.T., Tang, X.H.: Ground state solutions of Schröinger-Poisson systems with variable potential and convolution nonlinearity. J. Math. Anal. Appl. 73, 87-111 (2019) 
11. Chen, S.T., Tang, X.H.: Berestycki-Lions conditions on ground state solutions for a nonlinear Schrödinger equation with variable potentials. Adv. Nonlinear Anal. 9, 496-515 (2020)

12. Di Nezza, E., Palatucci, G., Valdinoci, E.: Hitchhiker's guide to the fractional Sobolev spaces. Bull. Sci. Math. 136, $521-573(2012)$

13. Dipierro, S., Palatucci, G., Valdinoci, E.: Existence and symmetry results for a Schrödinger type problem involving the fractional Laplacian. Matematiche 68(1), 201-216 (2013)

14. Fall, M.M., Valdinoci, E.: Uniqueness and nondegeneracy of positive solutions of $(-\Delta)^{s} u+u=u^{p}$ in $\mathbb{R}^{N}$ when $s$ is close to 1. Commun. Math. Phys. 329(1), 383-404 (2014)

15. Felmer, P., Quaas, A., Tan, J.: Positive solutions of the nonlinear Schrödinger equation with the fractional Laplacian. Proc. R. Soc. Edinb. A 142, 1237-1262 (2012)

16. Frank, R.L., Lenzmann, E.: Uniqueness and nondegeneracy of ground states for $(-\delta)^{s} Q+Q-Q^{\alpha+1}=0$ in $\mathbb{R}$. Acta Math. 210(2), 261-318 (2013)

17. Frank, R.L., Lenzmann, E., Silvestre, L.: Uniqueness of radial solutions for the fractional Laplacian. Commun. Pure Appl. Math. 69(9), 1671-1726 (2016)

18. Gao, Z., Tang, X.H., Chen, S.T.: Ground state solutions of fractional Choquard equations with general potentials and nonlinearities, Rev. R. Acad. Cienc. Exactas Fís. Nat., Ser. A Mat. 113 2037-2057 (2019)

19. Ground, X.J.C.: States of some fractional Schrödinger equations on $\mathbb{R}^{N}$. Proc. Edinb. Math. Soc. 58, 305-321 (2015)

20. Jeanjean, L.: On the existence of bounded Palais-Smale sequence and application to a Landesman-Lazer type problem set on $\mathbb{R}^{N}$. Proc. R. Soc. Edinb. A 129, 787-809 (1999)

21. Jeanjean, L., Tanaka, K.: A positive solution for a nonlinear Schrödinger equation on $\mathbb{R}^{N}$. Indiana Univ. Math. J. 54, 443-464 (2005)

22. Jeanjean, L., Toland, J.F.: Bounded Palais-Smale mountain-pass sequences. C. R. Acad. Sci. 327(1), 23-28 (1998)

23. Jin, H., Liu, W.B.: Ground state solutions for nonlinear fractional Schrödinger equations involving critical growth. Electron. J. Differ. Equ. 2017, 80 (2017)

24. Laskin, N.: Fractional Schrödinger equation. Phys. Rev. E 66, 056108 (2002)

25. Liu, Z.S., Ouyang, Z.G.: Existence of positive ground state solutions for fractional Schrödinger equations with a general nonlinearity. Appl. Anal. 97(7), 1154-1171 (2018)

26. Liu, Z.S., Squassina, M., Zhang, J.J.: Ground states for fractional Kirchhoff equations with critical nonlinearity in low dimension. Nonlinear Differ. Equ. Appl. 24, 50 (2017)

27. Papageorgiou, N.S., Rădulescu, V.D., Repovs, D.D.: Nonlinear Analysis-Theory and Methods. Springer Monographs in Mathematics. Springer, Berlin (2019)

28. Park, Y.J.: Fractional Polya-Szegö inequality. J. Chungcheong Math. Soc. 24(2), 267-271 (2011)

29. Rabinowitz, P: On a class of nonlinear Schrodinger equations. Z. Angew. Math. Phys. 43, 270-291 (1992)

30. Secchi, S.: On fractional Schrödinger equations in $\mathbb{R}^{N}$ without the Ambrosetti-Rabinowitz condition. arXiv preprint (2012). arXiv:1210.0755

31. Secchi, S.: Ground state solutions for nonlinear fractional Schrödinger equations in $\mathbb{R}^{N}$. J. Math. Phys. 54, 031501 (2013)

32. Tang, X.H., Chen, S.T.: Ground state solutions of Nehari-Pohozaev type for Kirchhoff-type problems with general potentials. Calc. Var. Partial Differ. Equ. 56, 110 (2017)

33. Tang, X.H., Chen, S.T.: Ground state solutions of Nehari-Pohozaev type for Schrödinger-Poisson problems with general potentials. Discrete Contin. Dyn. Syst. 37, 4973-5002 (2017)

34. Tang, X.H., Chen, S.T.: Singularly perturbed Choquard equations with nonlinearity satisfying Berestycki-Lions assumptions. Adv. Nonlinear Anal. 9, 413-437 (2020)

35. Tang, X.H., Chen, S.T.: Berestycki-Lions conditions on ground state solutions for a nonlinear Schrödinger equation with variable potentials. Adv. Nonlinear Anal. 9, 496-515 (2020)

36. Willem, M.: Minimax Theorems. Birkhäuser, Boston (1996)

37. Xiang, M.Q., Rădulescu, V.D., Zhang, B.L.: Combined effects for fractional Schrödinger-Kirchhoff systems with critical nonlinearities. ESAIM Control Optim. Calc. Var. 24, 1249-1273 (2018)

38. Xiang, M.Q., Rădulescu, V.D., Zhang, B.L.: Nonlocal Kirchhoff diffusion problems: local existence and blow-up of solutions. Nonlinearity 31, 3228-3250 (2018)

39. Xiang, M.Q., Rădulescu, V.D., Zhang, B.L.: Fractional Kirchhoff problems with critical Trudinger-Moser nonlinearity. Calc. Var. Partial Differ. Equ. 58, 57 (2019)

40. Xiang, M.Q., Rădulescu, V.D., Zhang, B.L.: A critical fractional Choquard-Kirchhoff problem with magnetic field. Commun. Contemp. Math. 21(4), 185004 (2019)

41. Yafaev, D.: Sharp constants in the Hardy-Rellich inequalities. J. Funct. Anal. 168(1), 121-144 (1999) 